Proc. Indian Acad. Sci. (Math. Sci.) Vol. 113, No. 3, August 2003, pp. 293-319.

Printed in India

\title{
Nonlinear second-order multivalued boundary value problems
}

\author{
LESZEK GASIŃSKI and NIKOLAOS S PAPAGEORGIOU* \\ Institute of Computer Science, Jagiellonian University, Nawojki 11, 30072 Cracow, \\ Poland \\ *Department of Mathematics, National Technical University, Zografou Campus, \\ Athens 15780, Greece \\ E-mail: gasinski@softlab.ii.uj.edu.pl; npapg@math.ntua.gr
}

MS received 21 June 2002

\begin{abstract}
In this paper we study nonlinear second-order differential inclusions involving the ordinary vector $p$-Laplacian, a multivalued maximal monotone operator and nonlinear multivalued boundary conditions. Our framework is general and unifying and incorporates gradient systems, evolutionary variational inequalities and the classical boundary value problems, namely the Dirichlet, the Neumann and the periodic problems. Using notions and techniques from the nonlinear operator theory and from multivalued analysis, we obtain solutions for both the 'convex' and 'nonconvex' problems. Finally, we present the cases of special interest, which fit into our framework, illustrating the generality of our results.
\end{abstract}

Keywords. Maximal monotone operator; pseudomonotone operator; Hartman condition; vector $p$-Laplacian; convex and nonconvex problems; Leray-Schauder alternative.

\section{Introduction}

In this paper we study the following nonlinear multivalued boundary value problem:

$$
\left\{\begin{array}{l}
\varphi\left(x^{\prime}(t)\right)^{\prime} \in A(x(t))+F(t, x(t)) \quad \text { a.e. on }[0, T] \\
\left(\varphi\left(x^{\prime}(0)\right),-\varphi\left(x^{\prime}(T)\right)\right) \in \xi(x(0), x(T)),
\end{array}\right.
$$

where $\varphi: \mathbb{R}^{N} \longrightarrow \mathbb{R}^{N}$ is the function defined by $\varphi(\zeta) \stackrel{d f}{=}\|\zeta\|_{\mathbb{R}^{N}}^{p-2} \zeta, p \geq 2, A: \mathbb{R}^{N} \supseteq$ $D(A) \longrightarrow 2^{\mathbb{R}^{N}}$ is a maximal monotone map, $F:[0, T] \times \mathbb{R}^{N} \longrightarrow 2^{\mathbb{R}^{N}}$ is a multivalued vector field and $\xi: \mathbb{R}^{N} \times \mathbb{R}^{N} \longrightarrow 2^{\mathbb{R}^{N} \times \mathbb{R}^{N}}$ is a maximal monotone map describing the boundary conditions.

We conduct a detailed study of problem (1.1) under the hypothesis that $F$ satisfies the Hartman condition (see [16] and p. 433 of [17]). Our formulation with the general nonlinear multivalued boundary conditions unifies the basic boundary value problems, namely the Dirichlet, Neumann and periodic boundary value problems, which can be obtained as special cases of problem (1.1) (see §5). Also the presence in 1.1) of the multivalued maximal monotone operator $A$, incorporates in our formulation second-order systems with nonsmooth convex potential. Moreover, since we also allow the possibility that the domain of $A$ is not all the $\mathbb{R}^{N}$ (i.e. $D(A)=\left\{\zeta \in \mathbb{R}^{N}: A(\zeta) \neq \emptyset\right\} \neq \mathbb{R}^{N}$ ), our study also includes second-order evolutionary variational inequalities. 
As we already mentioned, our basic hypothesis on $F$ is the so-called Hartman condition, which permits the derivation of a priori bounds for the solutions of 1.1. This condition was first employed by Hartman [16] for the vector Dirichlet problem

$$
\left\{\begin{array}{l}
x^{\prime \prime}=f(t, x) \\
x(0)=x(T)=0
\end{array}\right.
$$

where the function $f:[0, T] \times \mathbb{R}^{N} \longrightarrow \mathbb{R}^{N}$ is continuous. Later, it was used by Knobloch [24] for the vector periodic problem for a vector field which is locally Lipschitz in $\zeta \in \mathbb{R}^{N}$. Variants and extensions can be found in [13] and the references therein. Very recently the periodic problem was revisited by Mawhin [26], who used the vector $p$-Laplacian differential operator. Our work here extends this recent paper of Mawhin [26] in many different ways. We should point out that recently there has been an increasing interest for boundary value problems involving the $p$-Laplacian. However, the overwhelming majority of the works deal with the scalar problems (i.e. $N=1$ ) [2391422].

Boundary value problems for second-order differential inclusions were studied by Erbe and Krawcewicz [11], Frigon [12], Kandilakis and Papageorgiou [22] and Halidias and Papageorgiou [15]. In these papers $F$ depends also on $x^{\prime}$, but $A \equiv 0$ and the differential operator is the linear operator $x \longmapsto x^{\prime \prime}$. Recently, in a series of remarkable papers, De Blasi and Pianigiani [56778] developed the so-called 'Baire category method' for the derivation of strong relaxation results for the first-order multivalued Cauchy problems in separable Banach spaces.

Our approach is based on notions and results from multivalued analysis and from the theory of nonlinear operators of monotone type. We are led to an eventual application of a generalized version of the multivalued Leray-Schauder alternative principle, proved recently by Bader [1]. For the convenience of the reader, in the next section we recall some basic definitions and facts from these areas as well as the result of Bader, which will be used in the sequel. Our main references are [2021].

\section{Preliminaries}

Let $(\Omega, \Sigma)$ be a measurable space and let $X$ be a separable Banach space. We introduce the following notation:

$$
\begin{aligned}
& P_{f(c)}(X) \stackrel{d f}{=}\{A \subseteq X: A \text { is nonempty, closed (and convex) }\}, \\
& P_{(w) k(c)}(X) \stackrel{d f}{=}\{A \subseteq X: A \text { is nonempty, (weakly-) compact (and convex) }\} .
\end{aligned}
$$

A multifunction $F: \Omega \longrightarrow P_{f}(X)$ is said to be measurable, if for all $x \in X$, the function

$$
\Omega \ni \omega \longmapsto d(x, F(\omega)) \stackrel{d f}{=} \inf \left\{\|x-y\|_{X}: y \in F(\omega)\right\} \in \mathbb{R}_{+}^{N}
$$

is $\Sigma$-measurable. A multifunction $F: \Omega \longrightarrow 2^{X} \backslash\{\emptyset\}$ is said to be graph measurable, if

$$
\operatorname{Gr} F \stackrel{d f}{=}\{(\omega, x) \in \Omega \times X: x \in F(\omega)\} \in \Sigma \times \mathscr{B}(X),
$$


with $\mathscr{B}(X)$ being the Borel $\sigma$-field of $X$. For $P_{f}(X)$-valued multifunctions, measurability implies graph measurability, while the converse is true if $\Sigma$ is complete (i.e. $\Sigma=\widehat{\Sigma}=$ the universal $\sigma$-field). Recall that, if $\mu$ is a measure on $\Sigma$ and $\Sigma$ is $\mu$-complete, then $\Sigma=\widehat{\Sigma}$. Now, let $(\Omega, \Sigma, \mu)$ be a finite measure space. For a given multifunction $F: \Omega \longrightarrow 2^{X} \backslash\{\emptyset\}$ and $1 \leq p \leq+\infty$, we introduce the set

$$
S_{F}^{p} \stackrel{d f}{=}\left\{f \in L^{p}(\Omega ; X): f(\omega) \in F(\omega) \mu-\text { a.e. on } \Omega\right\} .
$$

In general, this set may be empty. It is easy to check that, if the map $\Omega \ni \omega \longmapsto \inf \left\{\|x\|_{X}\right.$ : $x \in F(\omega)\}$ is in $L^{p}(\Omega)$, then $S_{F}^{p} \neq \emptyset$.

Let $Y, Z$ be Hausdorff topological spaces. A multifunction $G: Y \longrightarrow 2^{Z} \backslash\{\emptyset\}$ is said to be lower semicontinuous (respectively upper semicontinuous), if for every closed set $C \subseteq Z$, the set $G^{+}(C) \stackrel{d f}{=}\{y \in Y: G(y) \subseteq C\}$ (respectively $G^{-}(C) \stackrel{d f}{=}\{y \in Y: G(y) \cap C \neq \emptyset\}$ ) is closed in $Y$. An upper semicontinuous multifunction with closed values has a closed graph (i.e. $\operatorname{Gr} G \stackrel{d f}{=}\{(y, z) \in Y \times Z: z \in G(y)\}$ is closed), while the converse is true if $G$ is locally compact (i.e. if for every $y \in Y$, there exists a neighbourhood $U$ of $y$ such that $\overline{G(U)}$ is compact in $Z$ ). Also, if $Z$ is a metric space, then $G$ is lower semicontinuous if and only if for every sequence $\left\{y_{n}\right\}_{n \geq 1} \subseteq Y$ such that $y_{n} \longrightarrow y$ in $Y$, we have

$$
G(y) \subseteq \liminf _{n \rightarrow+\infty} G\left(y_{n}\right)
$$

where

$$
\liminf _{n \rightarrow+\infty} G\left(y_{n}\right) \stackrel{d f}{=}\left\{z \in Z: \lim _{n \rightarrow+\infty} d\left(z, G\left(y_{n}\right)\right)=0\right\}
$$

or equivalently

$$
\liminf _{n \rightarrow+\infty} G\left(y_{n}\right) \stackrel{d f}{=}\left\{z \in Z: z=\lim _{n \rightarrow+\infty} z_{n} \text { where } z_{n} \in G\left(y_{n}\right), \text { for } n \geq 1\right\} .
$$

If $Z$ is a metric space, then on $P_{f}(Z)$ we can define a generalized metric $h$, known in the literature as the Hausdorff metric, by setting

$$
h(B, C) \stackrel{d f}{=} \max \left\{\sup _{b \in B} d(b, C), \sup _{c \in C} d(c, B)\right\} \quad \forall B, C \in P_{f}(Z) .
$$

If $Z$ is complete, then $\left(P_{f}(Z), h\right)$ is complete too. A multifunction $F: Y \longrightarrow P_{f}(Z)$ is said to be Hausdorff continuous (h-continuous for short), if it is continuous from $Y$ into $\left(P_{f}(Z), h\right)$.

Next, let $X$ be a reflexive Banach space and $X^{*}$ its topological dual. A map $A: X \supseteq$ $D(A) \longrightarrow 2^{X^{*}}$ is said to be monotone, if for all elements $\left(x, x^{*}\right),\left(y, y^{*}\right) \in \operatorname{Gr} A$, we have $\left\langle x^{*}-y^{*}, x-y\right\rangle \geq 0$ (by $\langle\cdot, \cdot\rangle$ we denote the duality brackets for the pair $\left(X, X^{*}\right)$ ). If additionally, the fact that $\left\langle x^{*}-y^{*}, x-y\right\rangle=0$ implies that $x=y$, then we say that $A$ is strictly monotone. The map $A$ is said to be maximal monotone, if it is monotone and the fact that $\left\langle x^{*}-y^{*}, x-y\right\rangle \geq 0$ for all $\left(x, x^{*}\right) \in \operatorname{Gr} A$, implies that $\left(y, y^{*}\right) \in \operatorname{Gr} A$. So, according to this definition, the graph of a maximal monotone map is maximal monotone with respect to inclusion among the graphs of all monotone maps from $X$ into $2^{X^{*}}$. It is easy to see that a maximal monotone map $A$ has a demiclosed graph, i.e., $\operatorname{Gr} A$ is sequentially closed in $X \times X_{w}^{*}$ and in $X_{w} \times X^{*}$ (here by $X_{w}$ and $X_{w}^{*}$, we denote the spaces $X$ and $X^{*}$ respectively, 
furnished with their weak topologies). If $A: X \longrightarrow X^{*}$ is everywhere defined and singlevalued, we say that $A$ is demicontinuous, if for every sequence $\left\{x_{n}\right\}_{n \geq 1} \subseteq X$ such that $x_{n} \longrightarrow x$ in $X$, we have that $A\left(x_{n}\right) \longrightarrow A(x)$ weakly in $X^{*}$. If map $A: X \longrightarrow X^{*}$ is monotone and demicontinuous, then it is also maximal monotone. A map $A: X \supseteq D(A) \longrightarrow 2^{X^{*}}$ is said to be coercive, if $D(A) \subseteq X$ is bounded or if $D(A)$ is unbounded and we have that

$$
\frac{\inf \left\{\left\langle x^{*}, x\right\rangle: x^{*} \in A(x)\right\}}{\|x\|_{X}} \longrightarrow+\infty \quad \text { as }\|x\|_{X} \rightarrow+\infty, \quad \text { with } x \in D(A) .
$$

A maximal monotone and coercive map is surjective.

If $H$ is a Hilbert space and $A: H \supseteq D(H) \longrightarrow 2^{H}$ is a maximal monotone map, then we can define the following well-known operators.

$$
\begin{array}{ll}
J_{\lambda} \stackrel{d f}{=}(I+\lambda A)^{-1} & (\text { the resolvent of } A), \\
A_{\lambda} \stackrel{d f}{=}=\frac{1}{\lambda}\left(I-J_{\lambda}\right) & (\text { the Yosida approximation of } A),
\end{array}
$$

for $\lambda>0$. Both these operators are single valued and everywhere defined. In addition, $J_{\lambda}$ is nonexpansive and $A_{\lambda}$ is monotone and Lipschitz continuous, with Lipschitz constant $1 / \lambda$ (hence $A_{\lambda}$ is maximal monotone). Moreover, if $A^{0}(x) \in A(x)$ is the unique element of minimum norm in $A(x)$ (i.e. $\left.\left\|A^{0}(x)\right\|_{H}=\min \left\{\|v\|_{H}: v \in A(x)\right\}\right)$, then

$$
\left\|A_{\lambda}(x)\right\|_{H} \leq\left\|A^{0}(x)\right\|_{H} \quad \forall x \in D(A), \lambda>0
$$

and

$$
A_{\lambda}(x) \longrightarrow A^{0}(x) \quad \text { in } H \text { as } \lambda \searrow 0 \forall x \in D(A) .
$$

Moreover

$$
\lim _{\lambda \searrow 0} J_{\lambda}(x)=\operatorname{proj}(x ; \overline{D(A)}) \quad \forall x \in H
$$

(here by $\operatorname{proj}(\cdot, \overline{D(A)})$ we denote the metric projection on $\bar{D}(A)$ and because $\overline{D(A)}$ is convex, since $A$ is maximal monotone, $\operatorname{proj}(\cdot, \overline{D(A)})$ is single valued). In particular, if $D(A)=H$, then $J_{\lambda} \longrightarrow I$ as $\lambda \searrow 0$, i.e., the resolvent is a kind of approximation of the identity on $H$. Note that, because $A$ is maximal monotone, for every $x \in D(A)$, the set $A(x)$ is nonempty, closed, convex and thus $A^{0}(x)$ is a well-defined unique vector in $A(x)$.

An operator $A: X \longrightarrow 2^{X^{*}}$ is said to be pseudomonotone, if

(a) for all $x \in X$, we have $A(x) \in P_{w k c}\left(X^{*}\right)$,

(b) $A$ is upper semicontinuous from every finite dimensional subspace $Z$ of $X$ into $X_{w}^{*}$,

(c) if $x_{n} \longrightarrow x$ weakly in $X, x_{n}^{*} \in A\left(x_{n}\right)$ and $\limsup _{n \rightarrow+\infty}\left\langle x_{n}^{*}, x_{n}-x\right\rangle \leq 0$, then for every $y \in X$, there exists $x^{*}(y) \in A(x)$, such that

$$
\left\langle x^{*}(y), x-y\right\rangle \leq \liminf _{n \rightarrow+\infty}\left\langle x_{n}^{*}, x_{n}-y\right\rangle .
$$

If $A$ is bounded (i.e. it maps bounded sets into bounded ones) and satisfies condition (c), then it satisfies condition (b) too. An operator $A: X \longrightarrow 2^{X^{*}}$ is said to be generalized pseudomonotone, if for all $x_{n}^{*} \in A\left(x_{n}\right)$, with $n \geq 1$, such that $x_{n} \longrightarrow x$ weakly in $X, x_{n}^{*} \longrightarrow$ $x^{*}$ weakly in $X^{*}$ and $\lim \sup _{n \rightarrow+\infty}\left\langle x_{n}^{*}, x_{n}-x\right\rangle \leq 0$, we have that

$$
x^{*} \in A(x) \quad \text { and } \quad\left\langle x_{n}^{*}, x_{n}\right\rangle \longrightarrow\left\langle x^{*}, x\right\rangle .
$$


Every maximal monotone operator is generalized pseudomonotone. Also a pseudomonotone operator is generalized pseudomonotone. The converse is true if the operator is everywhere defined and bounded. A pseudomonotone operator which is also coercive, is surjective.

Let $Y, Z$ be Banach spaces and let $K: Y \longrightarrow Z$ be a map. We say that $K$ is completely continuous, if the fact that $y_{n} \longrightarrow y$ weakly in $Y$ implies that $K\left(y_{n}\right) \longrightarrow K(y)$ in $Z$. We say that $K$ is compact, if it is continuous and maps bounded sets into relatively compact sets. In general, these two notions are distinct. However, if $Y$ is reflexive, then complete continuity implies compactness. Moreover, if $Y$ is reflexive and $K$ is linear, then the two notions are equivalent. Also a multifunction $F: Y \longrightarrow 2^{Z} \backslash\{\emptyset\}$ is said to be compact, if it is upper semicontinuous and maps bounded sets in $Y$ into relatively compact sets in $Z$.

As we already mentioned in the Introduction, our approach makes use of a generalization of the multivalued Leray-Schauder alternative principle (see [10], Theorem I.5.3, p. 61) due to [1] (see also [21], p. 346).

\section{PROPOSITION 1.}

If $Y, Z$ are two Banach spaces, $G: Y \longrightarrow P_{w k c}(Z)$ is upper semicontinuous into $Z_{w}, K$ : $Z \longrightarrow Y$ is completely continuous and $K \circ G$ is compact, then one of the following two statements holds: either (a) the set $S \stackrel{d f}{=}\{x \in Y: x \in \beta(K \circ G)(x)$ for some $0<\beta<1\} \subseteq Y$ is unbounded or (b) the map $K \circ G$ has a fixed point.

\section{Auxiliary results}

In this section we consider the following regularized version of 1.1 :

$$
\left\{\begin{array}{l}
\left(\varphi\left(x^{\prime}(t)\right)\right)^{\prime} \in A_{\lambda}(x(t))+F(t, x(t)) \quad \text { a.e. on }[0, T] \\
\left(\varphi\left(x^{\prime}(0)\right),-\varphi\left(x^{\prime}(T)\right)\right) \in \xi(x(0), x(T)),
\end{array}\right.
$$

with $\lambda>0$. Using Proposition 1 we will obtain a solution for problem 3.1. By a solution of 3.1 , we mean a function $x \in W^{1, p}\left([0, T] ; \mathbb{R}^{N}\right)$, such that $\left\|x^{\prime}(\cdot)\right\|_{\mathbb{R}^{N}}^{p-2} x^{\prime}(\cdot) \in$ $W^{1, p^{\prime}}\left([0, T] ; \mathbb{R}^{N}\right)$ (where $p \geq 2$ and $\left.1 / p+1 / p^{\prime}=1\right)$ and

$$
\left\{\begin{array}{l}
\left(\left\|x^{\prime}(t)\right\|_{\mathbb{R}^{N}}^{p-2} x^{\prime}(t)\right)^{\prime}=A_{\lambda}(x(t))+f(t) \quad \text { a.e. on }[0, T] \\
\left(\left\|x^{\prime}(0)\right\|_{\mathbb{R}^{N}}^{p-2} x^{\prime}(0),-\left\|x^{\prime}(T)\right\|_{\mathbb{R}^{N}}^{p-2} x^{\prime}(T)\right) \in \xi(x(0), x(T)),
\end{array}\right.
$$

where $f \in S_{F(\cdot, x(\cdot))}^{p^{\prime}}$. Recall that for $1<r<+\infty$, we have that the space $W^{1, r}\left([0, T] ; \mathbb{R}^{N}\right)$ is embedded continuously (in fact compactly) in $C\left([0, T] ; \mathbb{R}^{N}\right)$ and so the pointwise evaluation of $x$ and $\left\|x^{\prime}(\cdot)\right\|_{\mathbb{R}^{N}}^{p-2} x^{\prime}(\cdot)$ at $t=0$ and $t=T$ make sense. 
Our hypotheses on the data of (3.1) are the following:

$\mathrm{H}(\mathrm{A})_{1}: A: \mathbb{R}^{N} \supseteq D(A) \longrightarrow 2^{\mathbb{R}^{N}}$ is a maximal monotone map, such that $0 \in A(0)$.

$\mathrm{H}(\mathrm{F})_{1}: F:[0, T] \times \mathbb{R}^{N} \longrightarrow P_{k c}\left(\mathbb{R}^{N}\right)$ is a multifunction, such that

(i) for all $\zeta \in \mathbb{R}^{N}$, the multifunction $[0, T] \ni t \longmapsto F(t, \zeta) \in 2^{\mathbb{R}^{N}}$ is measurable;

(ii) for almost all $t \in[0, T]$, the multifunction $\mathbb{R}^{N} \ni \zeta \longmapsto F(t, \zeta) \in 2^{\mathbb{R}^{N}}$ has closed graph;

(iii) for all $k>0$ there exists $a_{k} \in L^{2}([0, T])_{+}$, such that for almost all $t \in[0, T]$, all $\zeta \in \mathbb{R}^{N}$ with $\|\zeta\|_{\mathbb{R}^{N}} \leq k$ and all $u \in F(t, \zeta)$, we have $\|u\|_{\mathbb{R}^{N}} \leq a_{k}(t)$;

(iv) there exists $M>0$, such that for almost all $t \in[0, T]$, all $\zeta \in \mathbb{R}^{N}$ with $\|\zeta\|_{\mathbb{R}^{N}}=M$ and all $u \in F(t, \zeta)$, we have $(u, \zeta)_{\mathbb{R}^{N}} \geq 0$ (Hartman condition).

$\mathrm{H}(\xi): \xi: \mathbb{R}^{N} \times \mathbb{R}^{N} \supseteq D(\xi): \longrightarrow 2^{\mathbb{R}^{N} \times \mathbb{R}^{N}}$ is a maximal monotone map, such that $(0,0) \in$ $\xi(0,0)$ and one of the following conditions holds:

(i) for every $\left(b, b^{\prime}\right) \in \xi\left(a, a^{\prime}\right)$, we have $(b, a)_{\mathbb{R}^{N}} \geq 0$ and $\left(b^{\prime}, a^{\prime}\right)_{\mathbb{R}^{N}} \geq 0$; or

(ii) $D(\xi)=\left\{\left(a, a^{\prime}\right) \in \mathbb{R}^{N} \times \mathbb{R}^{N}: a=a^{\prime}\right\}$.

Let

$$
\begin{gathered}
D \stackrel{d f}{=}\left\{x \in C^{1}\left([0, T] ; \mathbb{R}^{N}\right):\left\|x^{\prime}(\cdot)\right\|_{\mathbb{R}^{N}}^{p-2} x^{\prime}(\cdot) \in W^{1, p^{\prime}}\left([0, T] ; \mathbb{R}^{N}\right)\right. \text { and } \\
\left.\left(\left\|x^{\prime}(0)\right\|_{\mathbb{R}^{N}}^{p-2} x^{\prime}(0),-\left\|x^{\prime}(T)\right\|_{\mathbb{R}^{N}}^{p-2} x^{\prime}(T)\right) \in \xi(x(0), x(T))\right\}
\end{gathered}
$$

and let $V: L^{p}\left([0, T] ; \mathbb{R}^{N}\right) \supseteq D \longrightarrow L^{p^{\prime}}\left([0, T] ; \mathbb{R}^{N}\right)$ be defined by

$$
V(x)(\cdot) \stackrel{d f}{=}-\left(\left\|x^{\prime}(\cdot)\right\|_{\mathbb{R}^{N}}^{p-2} x^{\prime}(\cdot)\right)^{\prime} \quad \forall x \in D .
$$

With simple modifications in the proof of Proposition 3.1 of [22] (see also [15], proof of Theorem 1), we can have the following result:

\section{PROPOSITION 2.}

If hypothesis $\mathrm{H}(\xi)$ holds, then $V$ is maximal monotone.

Now we can state an existence result for problem 3.1.

\section{PROPOSITION 3.}

If hypotheses $\mathrm{H}(\mathrm{A})_{1}, \mathrm{H}(\mathrm{F})_{1}, \mathrm{H}(\xi)$ hold, then problem 3.1 has a solution $\bar{x} \in C^{1}\left([0, T] ; \mathbb{R}^{N}\right)$.

Proof. In what follows, by $\widehat{A_{\lambda}}: L^{p}\left([0, T] ; \mathbb{R}^{N}\right) \longrightarrow L^{p^{\prime}}\left([0, T] ; \mathbb{R}^{N}\right)$, we denote the Niemytzki operator corresponding to $A_{\lambda}$, i.e.

$$
\widehat{A}_{\lambda}(x)(\cdot)=A_{\lambda}(x(\cdot)) \quad \forall x \in L^{p}\left([0, T] ; \mathbb{R}^{N}\right) .
$$

Actually note that because $A_{\lambda}$ is Lipschitz continuous with constant $1 / \lambda$ and $A_{\lambda}(0)=0$, then

$$
\left\|A_{\lambda}(\zeta)\right\|_{\mathbb{R}^{N}} \leq \frac{1}{\lambda}\|\zeta\|_{\mathbb{R}^{N}} \quad \forall \zeta \in \mathbb{R}^{N}
$$


and so

$$
\widehat{A}_{\lambda}(x) \in L^{p}\left([0, T] ; \mathbb{R}^{N}\right) \subseteq L^{p^{\prime}}\left([0, T] ; \mathbb{R}^{N}\right) \quad \forall x \in L^{p}\left([0, T] ; \mathbb{R}^{N}\right)
$$

(since $2 \leq p<+\infty)$. Moreover, if $x \in C\left([0, T] ; \mathbb{R}^{N}\right)$, then $\widehat{A}_{\lambda}(x) \in C\left([0, T] ; \mathbb{R}^{N}\right)$. Also, let

$$
\widehat{\varphi}: L^{p}\left([0, T] ; \mathbb{R}^{N}\right) \longrightarrow L^{p^{\prime}}\left([0, T] ; \mathbb{R}^{N}\right)
$$

be the map, defined by

$$
\widehat{\varphi}(x)(\cdot) \stackrel{d f}{=}\|x(\cdot)\|_{\mathbb{R}^{N}}^{p-2} x(\cdot) \quad \forall x \in L^{p}\left([0, T] ; \mathbb{R}^{N}\right) .
$$

Both maps $\widehat{A}_{\lambda}$ and $\widehat{\varphi}$ are clearly continuous and monotone, thus maximal monotone. Let

$$
K_{\lambda} \stackrel{d f}{=} V+\widehat{A}_{\lambda}+\widehat{\varphi}: L^{p}\left([0, T] ; \mathbb{R}^{N}\right) \supseteq D \longrightarrow L^{p^{\prime}}\left([0, T] ; \mathbb{R}^{N}\right) .
$$

Claim 1. $K_{\lambda}$ is bijective.

From Proposition 2 and since $\widehat{A}_{\lambda}$ and $\widehat{\varphi}$ are both maximal monotone, it follows that $K_{\lambda}$ is maximal monotone too (see [20], Theorem III.3.3, p. 334). In what follows, by $\langle\cdot, \cdot\rangle_{p p^{\prime}}$, we denote the duality brackets for the pair $\left(L^{p}\left([0, T] ; \mathbb{R}^{N}\right), L^{p^{\prime}}\left([0, T] ; \mathbb{R}^{N}\right)\right)$. Because $\widehat{A}_{\lambda}(0)=0$, we have

$$
\left\langle K_{\lambda}(x), x\right\rangle_{p p^{\prime}} \geq\langle V(x), x\rangle_{p p^{\prime}}+\langle\widehat{\varphi}(x), x\rangle_{p p^{\prime}} \quad \forall x \in D .
$$

Using Green's identity (integration by parts), we obtain

$$
\begin{aligned}
\langle V(x), x\rangle_{p p^{\prime}}= & -\int_{0}^{T}\left(\left(\left\|x^{\prime}(t)\right\|_{\mathbb{R}^{N}}^{p-2} x^{\prime}(t)\right)^{\prime}, x(t)\right)_{\mathbb{R}^{N}} \mathrm{~d} t \\
= & -\left(\left\|x^{\prime}(T)\right\|_{\mathbb{R}^{N}}^{p-2} x^{\prime}(T), x(T)\right)_{\mathbb{R}^{N}}+\left(\left\|x^{\prime}(0)\right\|_{\mathbb{R}^{N}}^{p-2} x^{\prime}(0), x(0)\right)_{\mathbb{R}^{N}} \\
& +\int_{0}^{T}\left\|x^{\prime}(t)\right\|_{\mathbb{R}^{N}}^{p} \mathrm{~d} t .
\end{aligned}
$$

Because of hypotheses $\mathrm{H}(\xi)$, we know that

$$
-\left(\left\|x^{\prime}(T)\right\|_{\mathbb{R}^{N}}^{p-2} x^{\prime}(T), x(T)\right)_{\mathbb{R}^{N}}+\left(\left\|x^{\prime}(0)\right\|_{\mathbb{R}^{N}}^{p-2} x^{\prime}(0), x(0)\right)_{\mathbb{R}^{N}} \geq 0 .
$$

Therefore, we obtain

$$
\langle V(x), x\rangle_{p p^{\prime}} \geq\left\|x^{\prime}\right\|_{p}^{p} \quad \forall x \in D .
$$

Also $\langle\widehat{\varphi}(x), x\rangle_{p p^{\prime}}=\|x\|_{p}^{p}$. Hence

$$
\left\langle K_{\lambda}(x), x\right\rangle_{p p^{\prime}} \geq\left\|x^{\prime}\right\|_{p}^{p}+\|x\|_{p}^{p}=\|x\|_{1, p}^{p}
$$

(here by $\|\cdot\|_{1, p}$ we denote the norm in $W^{1, p}\left([0, T] ; \mathbb{R}^{N}\right)$ ). So we have proved that $K_{\lambda}$ is coercive. Recall that a maximal monotone and coercive operator is surjective. Also $\widehat{\varphi}$ is clearly strictly monotone and so we infer that $K_{\lambda}$ is injective and the claim is proved.

Thus, we can define the single valued operator

$$
K_{\lambda}^{-1}: L^{p^{\prime}}\left([0, T] ; \mathbb{R}^{N}\right) \longrightarrow L^{p}\left([0, T] ; \mathbb{R}^{N}\right) .
$$


Claim 2. $K_{\lambda}^{-1}: L^{p^{\prime}}\left([0, T] ; \mathbb{R}^{N}\right) \longrightarrow D \subseteq W^{1, p}\left([0, T] ; \mathbb{R}^{N}\right)$ is completely continuous (hence compact).

To this end, assume that

$$
y_{n} \longrightarrow y \quad \text { weakly in } L^{p^{\prime}}\left([0, T] ; \mathbb{R}^{N}\right),
$$

and set $x_{n} \stackrel{d f}{=} K_{\lambda}^{-1}\left(y_{n}\right)$, for $n \geq 1$, and $x \stackrel{d f}{=} K_{\lambda}^{-1}(y)$. For every $n \geq 1$, we have

$$
y_{n}=V\left(x_{n}\right)+\widehat{A}_{\lambda}\left(x_{n}\right)+\widehat{\varphi}\left(x_{n}\right)
$$

so

$$
\left\langle y_{n}, x_{n}\right\rangle_{p p^{\prime}}=\left\langle V\left(x_{n}\right), x_{n}\right\rangle_{p p^{\prime}}+\left\langle\widehat{A}_{\lambda}\left(x_{n}\right), x_{n}\right\rangle_{p p^{\prime}}+\left\langle\widehat{\varphi}\left(x_{n}\right), x_{n}\right\rangle_{p p^{\prime}}
$$

Recall that $\widehat{A}_{\lambda}(0)=0$ and $\widehat{A}_{\lambda}$ is maximal monotone and so we have that $\left\langle\widehat{A}_{\lambda}\left(x_{n}\right), x_{n}\right\rangle_{p p^{\prime}} \geq$ 0 . Hence, from 3.2, we have

$$
\left\|x_{n}\right\|_{1, p}^{p}=\left\|x_{n}\right\|_{p}^{p}+\left\|x_{n}^{\prime}\right\|_{p}^{p} \leq\left\langle y_{n}, x_{n}\right\rangle_{p p^{\prime}} \leq\left\|y_{n}\right\|_{p^{\prime}}\left\|x_{n}\right\|_{p} \leq\left\|y_{n}\right\|_{p^{\prime}}\left\|x_{n}\right\|_{1, p} .
$$

As the sequence $\left\{y_{n}\right\}_{n \geq 1} \subseteq L^{p^{\prime}}\left([0, T] ; \mathbb{R}^{N}\right)$ is bounded, so also the sequence $\left\{x_{n}\right\}_{n \geq 1} \subseteq$ $W^{1, p}\left([0, T] ; \mathbb{R}^{N}\right)$ is bounded. Thus, by passing to a subsequence if necessary, we may assume that

$$
x_{n} \longrightarrow z \quad \text { weakly in } W^{1, p}\left([0, T] ; \mathbb{R}^{N}\right)
$$

for some $z \in W^{1, p}\left([0, T] ; \mathbb{R}^{N}\right)$ and from the compactness of the embedding $W^{1, p}([0, T]$; $\left.\mathbb{R}^{N}\right) \subseteq L^{p}\left([0, T] ; \mathbb{R}^{N}\right)$, we also have that

$$
x_{n} \longrightarrow z \text { in } L^{p}\left([0, T] ; \mathbb{R}^{N}\right) .
$$

So, from 3.3 and the continuity of $\widehat{A}_{\lambda}$ and $\widehat{\varphi}$, we have that

$$
\begin{aligned}
& \left\langle y_{n}, x_{n}-z\right\rangle_{p p^{\prime}} \longrightarrow 0, \\
& \left\langle\widehat{A}_{\lambda}\left(x_{n}\right), x_{n}-z\right\rangle_{p p^{\prime}} \longrightarrow 0, \\
& \left\langle\widehat{\varphi}\left(x_{n}\right), x_{n}-z\right\rangle_{p p^{\prime}} \longrightarrow 0 .
\end{aligned}
$$

So, from 3.4, we obtain

$$
\lim _{n \rightarrow+\infty}\left\langle V\left(x_{n}\right), x_{n}-z\right\rangle_{p p^{\prime}}=0 .
$$

From 3.3 , we see that the sequence $\left\{V\left(x_{n}\right)\right\}_{n \geq 1} \subseteq L^{p^{\prime}}\left([0, T] ; \mathbb{R}^{N}\right)$ is bounded. So passing to a subsequence if necessary, we may have that

$$
V\left(x_{n}\right) \longrightarrow w \quad \text { weakly in } L^{p^{\prime}}\left([0, T] ; \mathbb{R}^{N}\right)
$$

for some $w \in L^{p^{\prime}}\left([0, T] ; \mathbb{R}^{N}\right)$. Because $V$ is maximal monotone (see Proposition [2), it is generalized pseudomonotone and $w=V(x)$, i.e.

$$
V\left(x_{n}\right) \longrightarrow V(z) \quad \text { weakly in } L^{p^{\prime}}\left([0, T] ; \mathbb{R}^{N}\right) .
$$


Thus exploiting the continuity of $\widehat{A}_{\lambda}$ and $\widehat{\varphi}$, in the limit as $n \rightarrow+\infty$, we obtain

$$
y=V(z)+\widehat{A}_{\lambda}(z)+\widehat{\varphi}(z)=K_{\lambda}(z)
$$

and so

$$
z=K_{\lambda}^{-1}(y)=x
$$

Moreover, from (3.5), we see that the sequence $\left\{\left\|x_{n}^{\prime}(\cdot)\right\|_{\mathbb{R}^{N}}^{p-2} x_{n}^{\prime}(\cdot)\right\}_{n \geq 1} \subseteq W^{1, p^{\prime}}\left([0, T] ; \mathbb{R}^{N}\right)$ is bounded and so, passing to a next subsequence if necessary, we may assume that

$$
\left\|x_{n}^{\prime}(\cdot)\right\|_{\mathbb{R}^{N}}^{p-2} x_{n}^{\prime}(\cdot) \longrightarrow g \quad \text { weakly in } W^{1, p^{\prime}}\left([0, T] ; \mathbb{R}^{N}\right)
$$

for some $g \in W^{1, p^{\prime}}\left([0, T] ; \mathbb{R}^{N}\right)$ and from the compactness of the embedding $W^{1, p^{\prime}}([0, T]$; $\left.\mathbb{R}^{N}\right) \subseteq C\left([0, T] ; \mathbb{R}^{N}\right)$, we have that

$$
\left\|x_{n}^{\prime}(\cdot)\right\|_{\mathbb{R}^{N}}^{p-2} x_{n}^{\prime}(\cdot) \longrightarrow g \quad \text { in } C\left([0, T] ; \mathbb{R}^{N}\right) .
$$

So

$$
\left\|x_{n}^{\prime}(t)\right\|_{\mathbb{R}^{N}}^{p-2} x_{n}^{\prime}(t) \longrightarrow g(t) \quad \forall t \in[0, T]
$$

and because the function $\varphi: \mathbb{R}^{N} \ni \zeta \longmapsto\|\zeta\|_{\mathbb{R}^{N}}^{p-2} \zeta \in \mathbb{R}^{N}$ is a homeomorphism, we also have $x_{n}^{\prime}=\varphi^{-1}\left(\varphi\left(x_{n}^{\prime}(\cdot)\right)\right) \longrightarrow \varphi^{-1}(g(\cdot))$ and so

$$
x_{n}^{\prime} \longrightarrow \varphi^{-1}(g(\cdot)) \quad \text { weakly in } L^{p}\left([0, T] ; \mathbb{R}^{N}\right) .
$$

Because $x_{n} \longrightarrow x$ weakly in $W^{1, p}\left([0, T] ; \mathbb{R}^{N}\right)$, it follows that $x^{\prime}(\cdot)=\varphi^{-1}(g(\cdot))$. Hence $g(t)=\left\|x^{\prime}(t)\right\|_{\mathbb{R}^{N}}^{p-2} x^{\prime}(t)$ for almost all $t \in[0, T]$. Therefore

$$
\varphi\left(x_{n}^{\prime}(\cdot)\right) \longrightarrow \varphi\left(x^{\prime}(\cdot)\right) \quad \text { in } C\left([0, T] ; \mathbb{R}^{N}\right)
$$

and so

$$
x_{n}^{\prime} \longrightarrow x^{\prime} \quad \text { in } L^{p}\left([0, T] ; \mathbb{R}^{N}\right) .
$$

Thus, we have proved that

$$
x_{n} \longrightarrow x \text { in } W^{1, p}\left([0, T] ; \mathbb{R}^{N}\right) .
$$

Since every subsequence of $\left\{x_{n}\right\} \subseteq W^{1, p}\left([0, T] ; \mathbb{R}^{N}\right)$ has a further subsequence converging strongly to $x$ in $W^{1, p}\left([0, T] ; \mathbb{R}^{N}\right)$, we infer that the whole sequence converges strongly to $x$ and this proves the complete continuity of $K_{\lambda}^{-1}$. Moreover, since $L^{p^{\prime}}\left([0, T] ; \mathbb{R}^{N}\right)$ is reflexive, $K_{\lambda}^{-1}$ is also compact. Thus, the proof of Claim 2 is complete.

Consider the following modification of the oriented field $F(t, \zeta)$ :

$$
F_{1}(t, \zeta)=-F\left(t, p_{M}(\zeta)\right)+\varphi\left(p_{M}(\zeta)\right)
$$


where $M>0$ is as in hypothesis $\mathrm{H}(\mathrm{F})_{1}(\mathrm{iv})$ and $p_{M}: \mathbb{R}^{N} \longrightarrow \mathbb{R}^{N}$ is the $M$-radial retraction defined by

$$
p_{M}(\zeta) \stackrel{d f}{=} \begin{cases}\zeta & \text { if } \quad\|\zeta\|_{\mathbb{R}^{N}} \leq M \\ \frac{M \zeta}{\|\zeta\|_{\mathbb{R}^{N}}} & \text { if }\|\zeta\|_{\mathbb{R}^{N}}>M .\end{cases}
$$

Evidently, $F_{1}(t, \zeta)$ is measurable in $t \in[0, T]$, has closed graph in $\zeta \in \mathbb{R}^{N}$ and for almost all $t \in[0, T]$, all $\zeta \in \mathbb{R}^{N}$ and all $u \in F_{1}(t, \zeta)$, we have that $\|u\|_{\mathbb{R}^{N}} \leq \bar{a}_{M}(t)$ for some $\bar{a}_{M} \in$ $L^{2}(0, T)_{+}$, namely $\bar{a}_{M} \stackrel{d f}{=} a_{M}+M^{p-1}$ (see hypothesis $\mathrm{H}(\mathrm{F})_{1}(\mathrm{iii})$ ). Consider the multivalued Niemytzki operator

$$
\widehat{F}_{1}: L^{p}\left([0, T] ; \mathbb{R}^{N}\right) \longrightarrow 2^{L^{p^{\prime}}\left([0, T] ; \mathbb{R}^{N}\right)}
$$

corresponding to $F_{1}$ and defined by

$$
\widehat{F}_{1}(x) \stackrel{d f}{=} S_{F_{1}(\cdot, x(\cdot))}^{p^{\prime}} \quad \forall x \in L^{p}\left([0, T] ; \mathbb{R}^{N}\right) .
$$

Using the properties of $F_{1}$, as in the proof of Theorem 3 of [15], we can show that $\widehat{F}_{1}$ is $P_{w k c}\left(L^{p^{\prime}}\left([0, T] ; \mathbb{R}^{N}\right)\right)$-valued and the multifunction $x \longmapsto \widehat{F}_{1}(x)$ is upper semicontinuous from $L^{p}\left([0, T] ; \mathbb{R}^{N}\right)$ into $L^{p^{\prime}}\left([0, T] ; \mathbb{R}^{N}\right)_{w}$ and so also from the space $W^{1, p}\left([0, T] ; \mathbb{R}^{N}\right)$ into the space $L^{p^{\prime}}\left([0, T] ; \mathbb{R}^{N}\right)_{w}$.

Consider the multivalued map

$$
K_{\lambda}^{-1} \circ \widehat{F}_{1}: W^{1, p}\left([0, T] ; \mathbb{R}^{N}\right) \longrightarrow P_{k}\left(W^{1, p}\left([0, T] ; \mathbb{R}^{N}\right)\right)
$$

(see Claim 2). We want to obtain a fixed point for the operator $K_{\lambda}^{-1} \circ \widehat{F}_{1}$, by using Proposition 1 For this purpose, let

$$
S \stackrel{d f}{=}\left\{x \in W^{1, p}\left([0, T] ; \mathbb{R}^{N}\right): x \in \beta\left(K_{\lambda}^{-1} \circ \widehat{F}_{1}\right)(x), 0<\beta<1\right\} .
$$

Claim 3. The set $S \subseteq W^{1, p}\left([0, T] ; \mathbb{R}^{N}\right)$ is bounded.

Let $x \in S$. We have

$$
K_{\lambda}\left(\frac{1}{\beta} x\right) \in \widehat{F}_{1}(x) \quad \forall \beta \in(0,1) .
$$

So

$$
V\left(\frac{1}{\beta} x\right)+\widehat{A}_{\lambda}\left(\frac{1}{\beta} x\right)+\widehat{\varphi}\left(\frac{1}{\beta} x\right) \in \widehat{F}_{1}(x) \quad \forall \beta \in(0,1) .
$$

Thus

$$
\begin{aligned}
& \left\langle V\left(\frac{1}{\beta} x\right), x\right\rangle_{p p^{\prime}}+\left\langle\widehat{A}_{\lambda}\left(\frac{1}{\beta} x\right), x\right\rangle_{p p^{\prime}}+\left\langle\widehat{\varphi}\left(\frac{1}{\beta} x\right), x\right\rangle_{p p^{\prime}} \\
& =-\langle f, x\rangle_{p p^{\prime}}+\left\langle\hat{\varphi}\left(p_{M}(x(\cdot))\right), x\right\rangle_{p p^{\prime}},
\end{aligned}
$$


with $f \in S_{F\left(\cdot, p_{M}(x(\cdot))\right)}^{p^{\prime}}$. As before, we have that

$$
\left\langle\widehat{A}_{\lambda}\left(\frac{1}{\beta} x\right), x\right\rangle_{p p^{\prime}} \geq 0 \quad \forall \beta \in(0,1) .
$$

Moreover, using Green's indentity and hypothesis $\mathrm{H}(\xi)$, we obtain

$$
\left\langle V\left(\frac{1}{\beta} x\right), x\right\rangle_{p p^{\prime}} \geq \frac{1}{\beta^{p-1}}\left\|x^{\prime}\right\|_{p}^{p}
$$

Also, we have

$$
\left\langle\widehat{\varphi}\left(\frac{1}{\beta} x\right), x\right\rangle_{p p^{\prime}} \geq \frac{1}{\beta^{p-1}}\|x\|_{p}^{p} .
$$

Using these inequalities in (3.6) and applying hypothesis $\mathrm{H}(\mathrm{F})_{1}$ (iii) (recall that $f(t) \in$ $-F\left(t, p_{M}(x(t))\right)$ almost everywhere on $[0, T]$ and so $\|f(t)\|_{\mathbb{R}^{N}} \leq a_{M}(t)$ almost everywhere on $[0, T]$, we obtain

$$
\begin{aligned}
& \frac{1}{\beta^{p-1}}\|x\|_{p}^{p}+\frac{1}{\beta^{p-1}}\left\|x^{\prime}\right\|_{p}^{p} \\
& \quad \leq\|f\|_{p^{\prime}}\|x\|_{p}+\left\langle\left\|p_{M}(x(\cdot))\right\|_{\mathbb{R}^{N}}^{p-2} p_{M}(x(\cdot)), x(\cdot)\right\rangle_{p p^{\prime}} \\
& \quad \leq\left\|a_{M}\right\|_{p^{\prime}}\|x\|_{p}+\int_{\{x>M\}} M^{p-1}\|x(t)\|_{\mathbb{R}^{N}} \mathrm{~d} t+\int_{\{x \leq M\}}\|x(t)\|_{\mathbb{R}^{N}}^{p} \mathrm{~d} t \\
& \quad \leq T^{\left(2-p^{\prime}\right) / 2 p^{\prime}}\left\|a_{M}\right\|_{2}\|x\|_{p}+M^{p-1}\|x\|_{1}+\|x\|_{p}^{p} .
\end{aligned}
$$

As $\left(1 / \beta^{p-1}\right)>1$, we have

$$
\|x\|_{1, p}^{p} \leq\left(T^{\left(2-p^{\prime}\right) / 2 p^{\prime}}\left\|a_{M}\right\|_{2}+M^{p-1} T^{(p-1) / p}\right)\|x\|_{p} .
$$

Thus, we conclude that

$$
\|x\|_{1, p} \leq c_{1},
$$

for some $c_{1}>0$ independent on $x \in S$. This proves Claim 3 .

Now we can apply Proposition $\square$ and obtain $\bar{x} \in D$, such that $\bar{x} \in\left(K_{\lambda}^{-1} \circ \widehat{F}_{1}\right)(\bar{x})$.

Claim 4. For all $t \in[0, T]$, we have that $\|\bar{x}(t)\|_{\mathbb{R}^{N}} \leq M$.

Suppose, that the claim is not true. Then, we can find $t_{1}, t_{2} \in[0, T]$, with $t_{1}<t_{2}$, such that

$$
\left\|\bar{x}\left(t_{1}\right)\right\|_{\mathbb{R}^{N}}=M \text { or } t_{1}=0
$$

and

$$
\left\|\bar{x}\left(t_{2}\right)\right\|_{\mathbb{R}^{N}}=\max _{t \in[0, T]}\|\bar{x}(t)\|_{\mathbb{R}^{N}}>M
$$


and

$$
\|\bar{x}(t)\|_{\mathbb{R}^{N}}>M \quad \forall t \in\left(t_{1}, t_{2}\right] .
$$

Since $\bar{x} \in\left(K_{\lambda}^{-1} \circ \widehat{F}_{1}\right)(\bar{x})$, we have that

$$
K_{\lambda}(\bar{x})=\widehat{F}_{1}(\bar{x})
$$

and so

$$
\left(\left\|\bar{x}^{\prime}(t)\right\|_{\mathbb{R}^{N}}^{p-2} \bar{x}^{\prime}(t)\right)^{\prime}=A_{\lambda}(\bar{x}(t))+f(t)+\varphi(\bar{x}(t))-\varphi\left(p_{M}(\bar{x}(t))\right)
$$

almost everywhere on $[0, \mathrm{~T}]$, with $\bar{x} \in D$ and $f \in S_{F\left(\cdot, p_{M}(\bar{x}(\cdot))\right)}^{p^{\prime}}$. Then, for almost all $t \in$ $\left(t_{1}, t_{2}\right]$, we have

$$
\begin{aligned}
& \frac{\mathrm{d}}{\mathrm{d} t}\left(\left\|\bar{x}^{\prime}(t)\right\|_{\mathbb{R}^{N}}^{p-2} \bar{x}^{\prime}(t), \bar{x}(t)\right)_{\mathbb{R}^{N}} \\
& \quad=\left(\left(\left\|\bar{x}^{\prime}(t)\right\|_{\mathbb{R}^{N}}^{p-2} \bar{x}^{\prime}(t)\right)^{\prime}, \bar{x}(t)\right)_{\mathbb{R}^{N}}+\left(\left\|\bar{x}^{\prime}(t)\right\|_{\mathbb{R}^{N}}^{p-2} \bar{x}^{\prime}(t), \bar{x}^{\prime}(t)\right)_{\mathbb{R}^{N}} \\
& =\left(A_{\lambda}(\bar{x}(t))+f(t)+\varphi(\bar{x}(t))-\varphi\left(p_{M}(\bar{x}(t))\right), \bar{x}(t)\right)_{\mathbb{R}^{N}}+\left\|\bar{x}^{\prime}(t)\right\|_{\mathbb{R}^{N}}^{p} \\
& \quad \geq \frac{\|\bar{x}(t)\|_{\mathbb{R}^{N}}}{M}\left(f(t), p_{M}(\bar{x}(t))\right)_{\mathbb{R}^{N}}+\|\bar{x}(t)\|_{\mathbb{R}^{N}}^{p}-M^{p-1}\|\bar{x}(t)\|_{\mathbb{R}^{N}} .
\end{aligned}
$$

By virtue of hypothesis $\mathrm{H}(\mathrm{F})_{1}$ (iv), we have that

$$
\frac{\|\bar{x}(t)\|_{\mathbb{R}^{N}}}{M}\left(f(t), p_{M}(\bar{x}(t))\right)_{\mathbb{R}^{N}} \geq 0 \quad \text { a.e. on }\left(t_{1}, t_{2}\right]
$$

and so we obtain that

$$
\frac{\mathrm{d}}{\mathrm{d} t}\left(\left\|\bar{x}^{\prime}(t)\right\|_{\mathbb{R}^{N}}^{p-2} \bar{x}^{\prime}(t), \bar{x}(t)\right)_{\mathbb{R}^{N}} \geq\|\bar{x}(t)\|_{\mathbb{R}^{N}}\left(\|\bar{x}(t)\|_{\mathbb{R}^{N}}^{p-1}-M^{p-1}\right)>0
$$

almost everywhere on $\left(t_{1}, t_{2}\right]$.

Suppose that $0<t_{2}<T$. Then for $r(t) \stackrel{d f}{=}\|x(t)\|_{\mathbb{R}^{N}}^{2}$, we have $r^{\prime}\left(t_{2}\right)=0$ and so $\left(x^{\prime}\left(t_{2}\right), x\left(t_{2}\right)\right)_{\mathbb{R}^{N}}=0$. From (3.7), we have that the function

$$
\left(t_{1}, t_{2}\right] \ni t \longmapsto\left(\left\|\bar{x}^{\prime}(t)\right\|_{\mathbb{R}^{N}}^{p-2} \bar{x}^{\prime}(t), \bar{x}(t)\right)_{\mathbb{R}^{N}} \in \mathbb{R}
$$

is strictly increasing. This means that

$$
\left\|\bar{x}^{\prime}(t)\right\|_{\mathbb{R}^{N}}^{p-2}\left(\bar{x}^{\prime}(t), \bar{x}(t)\right)_{\mathbb{R}^{N}}<\left\|\bar{x}^{\prime}\left(t_{2}\right)\right\|_{\mathbb{R}^{N}}^{p-2}\left(\bar{x}^{\prime}\left(t_{2}\right), \bar{x}\left(t_{2}\right)\right)_{\mathbb{R}^{N}} \quad \forall t \in\left[t_{1}, t_{2}\right) .
$$

So

$$
\left(\bar{x}^{\prime}(t), \bar{x}(t)\right)_{\mathbb{R}^{N}}=\frac{1}{2} r^{\prime}(t)<0 \quad \forall t \in\left[t_{1}, t_{2}\right) .
$$


Thus

$$
M^{2}<\left\|\bar{x}\left(t_{2}\right)\right\|_{\mathbb{R}^{N}}^{2}<\left\|\bar{x}\left(t_{1}\right)\right\|_{\mathbb{R}^{N}}^{2}
$$

what is a contradition with $\left\|\bar{x}\left(t_{2}\right)\right\|_{\mathbb{R}^{N}}=\max _{t \in[0, T]}\|\bar{x}(t)\|_{\mathbb{R}^{N}} \geq\left\|\bar{x}\left(t_{1}\right)\right\|_{\mathbb{R}^{N}}$.

Suppose that $t_{2}=T$. Then $r^{\prime}(T) \geq 0$. On the other hand, if hypothesis $\mathrm{H}(\xi)(\mathrm{i})$ is in effect, we have

$$
\left(-\left\|\bar{x}^{\prime}(T)\right\|_{\mathbb{R}^{N}}^{p-2} \bar{x}^{\prime}(T), \bar{x}(T)\right)_{\mathbb{R}^{N}} \geq 0 .
$$

Hence $\left(\bar{x}^{\prime}(T), \bar{x}(T)\right)_{\mathbb{R}^{N}} \leq 0$ and so $r^{\prime}(T)=0$ and we argue as before. If hypothesis $\mathrm{H}(\xi)$ (ii) is in effect, then $\bar{x}(T)=\bar{x}(0)$ and $r^{\prime}(0) \leq 0 \leq r^{\prime}(T)$. As $(0,0) \in \xi(0,0)$ and $\xi$ is maximal monotone, from inclusion in 3.1), we conclude that $\left(x^{\prime}(0), x(0)\right)_{\mathbb{R}^{N}} \geq$ $\left(x^{\prime}(T), x(T)\right)_{\mathbb{R}^{N}}$ and thus $r^{\prime}(0) \geq r^{\prime}(T)$. So, we have that $r^{\prime}(0)=r^{\prime}(T)=0$ and next we argue as above. Finally, if $t_{2}=0$, than $t_{1}=t_{2}=0$ and the claim is automatically true. This proves Claim 4.

Because of Claim 4, for a fixed point $\bar{x} \in D$ of $K_{\lambda}^{-1} \circ \widehat{F}_{1}$, we have that $p_{M}(\bar{x}(t))=\bar{x}(t)$ for all $t \in[0, T]$ and therefore

$$
\left\{\begin{array}{l}
\left(\left\|\bar{x}^{\prime}(t)\right\|_{\mathbb{R}^{N}}^{p-2} \bar{x}^{\prime}(t)\right)^{\prime} \in A_{\lambda}(\bar{x}(t))+F(t, \bar{x}(t)) \quad \text { a.e. on }[0, T] \\
\left(\varphi\left(\bar{x}^{\prime}(0)\right),-\varphi\left(\bar{x}^{\prime}(T)\right)\right) \in \xi(\bar{x}(0), \bar{x}(T)),
\end{array}\right.
$$

i.e. $\bar{x} \in C^{1}\left([0, T] ; \mathbb{R}^{N}\right)$ is a solution of 3.1 .

\section{Existence theorems}

In this section we prove existence theorems for problem (1.1) under different hypotheses on $A$ and $F$. First we examine the case where $\operatorname{dom} A \neq \mathbb{R}^{N}$ (hypothesis $\mathrm{H}(\mathrm{A})_{1}$ ) and $F$ is convex valued (hypothesis $\mathrm{H}(\mathrm{F})_{1}-$ convex problem). Then we assume that $\operatorname{dom} A=$ $\mathbb{R}^{N}$ (see hypothesis $\mathrm{H}(\mathrm{A})_{2}$ below) and this allows us to slightly generalize the growth condition on $F$ (see hypothesis $\mathrm{H}(\mathrm{F})_{2}$ ). Finally for both cases $\operatorname{dom} A \neq \mathbb{R}^{N}$ and $\operatorname{dom} A=$ $\mathbb{R}^{N}$, we consider the 'nonconvex problem' (i.e. $F$ does not need to have convex values, see hypotheses $\mathrm{H}(\mathrm{F})_{3}$ and $\left.\mathrm{H}(\mathrm{F})_{4}\right)$.

To have an existence theorem for problem (1.1), we need a hypothesis that relates the monotonicity term $A$ of the inclusion with the monotone term $\xi$ of the boundary conditions.

$\mathrm{H}_{0}$ : for all $\lambda>0$, all $\left(a, a^{\prime}\right) \in D(\xi)$ and all $\left(b, b^{\prime}\right) \in \xi\left(a, a^{\prime}\right)$, we have

$$
\left(A_{\lambda}(a), b\right)_{\mathbb{R}^{N}}+\left(A_{\lambda}\left(a^{\prime}\right), b^{\prime}\right)_{\mathbb{R}^{N}} \geq 0 .
$$

Remark 1. If $\xi=\partial \psi$ with $\psi: \mathbb{R}^{N} \times \mathbb{R}^{N} \longrightarrow \mathbb{R}$ convex (hence locally Lipschitz), then if by $\partial_{i} \psi$, for $i=1,2$, we denote the partial subdifferential of $\psi\left(a, a^{\prime}\right)$ with respect to $a$ and $a^{\prime}$ respectively, then we know that

$$
\partial \psi\left(a, a^{\prime}\right) \subseteq \partial_{1} \psi\left(a, a^{\prime}\right) \times \partial_{2} \psi\left(a, a^{\prime}\right)
$$


Remark 2. In this setting, we know that

$$
\left(A_{\lambda}(a), b\right)_{\mathbb{R}^{N}} \geq 0 \text { and }\left(A_{\lambda}\left(a^{\prime}\right), b^{\prime}\right)_{\mathbb{R}^{N}} \geq 0 \forall\left(a, a^{\prime}\right) \in D(\xi),\left(b, b^{\prime}\right) \in \xi\left(a, a^{\prime}\right),
$$

Remark 3. is equivalent to saying that

$$
\psi\left(J_{\lambda}(a), a^{\prime}\right) \leq \psi\left(a, a^{\prime}\right) \text { and } \psi\left(a, J_{\lambda}\left(a^{\prime}\right)\right) \leq \psi\left(a, a^{\prime}\right) \text { respectively, }
$$

(see [20]).

Theorem 1. If hypotheses $\mathrm{H}(\mathrm{A})_{1}, \mathrm{H}(\mathrm{F})_{1}, \mathrm{H}(\xi)$ and $\mathrm{H}_{0}$ hold, then problem 1.1. has at least one solution $\bar{x} \in C^{1}\left([0, T] ; \mathbb{R}^{N}\right)$.

Proof. Let $\lambda_{n} \searrow 0$ and let $x_{n} \in C\left([0, T] ; \mathbb{R}^{N}\right)$ be solutions of the corresponding problem 3.1 with $\lambda=\lambda_{n}$, for $n \geq 1$. Such solutions exist by virtue of Proposition 3 Moreover, from the proof of Proposition 3 (see Claim 4), we know that

$$
\left\|x_{n}\right\|_{\infty} \leq M \quad \forall n \geq 1,
$$

with $M>0$ as in hypothesis $\mathrm{H}(\mathrm{F})_{1}$ (iv). We have

$$
V\left(x_{n}\right)+\widehat{A}_{\lambda_{n}}\left(x_{n}\right)=-f_{n} \quad \text { with } f_{n} \in S_{F\left(\cdot, x_{n}(\cdot)\right)}^{p^{\prime}} .
$$

So

$$
\left\langle V\left(x_{n}\right), x_{n}\right\rangle_{p p^{\prime}}+\left\langle\widehat{A}_{\lambda_{n}}\left(x_{n}\right), x_{n}\right\rangle_{p p^{\prime}}=-\left\langle f_{n}, x_{n}\right\rangle_{p p^{\prime}}
$$

We know that $\left\langle\widehat{A}_{\lambda_{n}}\left(x_{n}\right), x_{n}\right\rangle_{p p^{\prime}} \geq 0$ and as before via Green's identity and since $x_{n} \in D$, we have that

$$
\left\|x_{n}^{\prime}\right\|_{p}^{p} \leq\left\langle V\left(x_{n}\right), x_{n}\right\rangle_{p p^{\prime}} .
$$

So, from hypothesis $\mathrm{H}(\mathrm{F})_{1}(\mathrm{iii})$, we have

$$
\left\|x_{n}^{\prime}\right\|_{p}^{p} \leq\left\|f_{n}\right\|_{p^{\prime}}\left\|x_{n}\right\|_{p} \leq T^{1 / p} M\left\|a_{M}\right\|_{p^{\prime}} \quad \forall n \geq 1 .
$$

Thus the sequence $\left\{x_{n}^{\prime}\right\}_{n \geq 1} \subseteq L^{p}\left([0, T] ; \mathbb{R}^{N}\right)$ is bounded and from 4.1], also $\left\{x_{n}\right\}_{n \geq 1} \subseteq$ $W^{1, p}\left([0, T] ; \mathbb{R}^{N}\right)$ is bounded. Hence, by passing to a subsequence if necessary, we may assume that

$$
x_{n} \longrightarrow \bar{x} \quad \text { weakly in } W^{1, p}\left([0, T] ; \mathbb{R}^{N}\right) .
$$

Note that $\widehat{A}_{\lambda}\left(x_{n}\right) \in C\left([0, T] ; \mathbb{R}^{N}\right)$ and because of hypothesis $\mathrm{H}(\mathrm{F})_{1}$ (iii), we have that $\left\{f_{n}\right\}_{n \geq 1} \subseteq L^{2}\left([0, T] ; \mathbb{R}^{N}\right) \subseteq L^{p^{\prime}}\left([0, T] ; \mathbb{R}^{N}\right)$. So we obtain

$$
\left\langle V\left(x_{n}\right), \widehat{A}_{\lambda_{n}}\left(x_{n}\right)\right\rangle_{p p^{\prime}}+\left\|\widehat{A}_{\lambda_{n}}\left(x_{n}\right)\right\|_{2}^{2}=-\left\langle f_{n}, \widehat{A}_{\lambda_{n}}\left(x_{n}\right)\right\rangle_{p p^{\prime}} .
$$


From Green's identity, we have that

$$
\begin{aligned}
\left\langle V\left(x_{n}\right), \widehat{A}_{\lambda_{n}}\left(x_{n}\right)\right\rangle_{p p^{\prime}}= & -\int_{0}^{T}\left(\left(\left\|x_{n}^{\prime}(t)\right\|_{\mathbb{R}^{N}}^{p-2} x_{n}^{\prime}(t)\right)^{\prime}, A_{\lambda_{n}}\left(x_{n}(t)\right)\right)_{\mathbb{R}^{N}} \mathrm{~d} t \\
= & -\left(\left\|x_{n}^{\prime}(T)\right\|_{\mathbb{R}^{N}}^{p-2} x_{n}^{\prime}(T), A_{\lambda_{n}}\left(x_{n}(T)\right)\right)_{\mathbb{R}^{N}} \\
& +\left(\left\|x_{n}^{\prime}(0)\right\|_{\mathbb{R}^{N}}^{p-2} x_{n}^{\prime}(0), A_{\lambda_{n}}\left(x_{n}(0)\right)\right)_{\mathbb{R}^{N}} \\
& +\int_{0}^{T}\left(\left\|x_{n}^{\prime}(t)\right\|_{\mathbb{R}^{N}}^{p-2} x_{n}^{\prime}(t), \frac{\mathrm{d}}{\mathrm{d} t} A_{\lambda_{n}}\left(x_{n}(t)\right)\right)_{\mathbb{R}^{N}} \mathrm{~d} t .
\end{aligned}
$$

Recall that the Yosida approximation $A_{\lambda_{n}}: \mathbb{R}^{N} \longrightarrow \mathbb{R}^{N}$ is Lipschitz continuous and so by Rademacher's theorem, it is differentiable almost everywhere. Also $A_{\lambda_{n}}$ is monotone. If $\zeta \in \mathbb{R}^{N}$ is a point of differentiability of $A_{\lambda_{n}}$, then from the monotonicity property, we have

$$
\left(\zeta^{\prime}, \frac{A_{\lambda_{n}}\left(\zeta+t \zeta^{\prime}\right)-A_{\lambda_{n}}(\zeta)}{t}\right)_{\mathbb{R}^{N}} \geq 0 \quad \forall t>0, \zeta^{\prime} \in \mathbb{R}^{N}
$$

So passing to the limit as $t \rightarrow 0$, we have

$$
\left(\zeta^{\prime}, A_{\lambda_{n}}^{\prime}(\zeta) \zeta^{\prime}\right)_{\mathbb{R}^{N}} \geq 0
$$

From the chain rule of Marcus and Mizel [25], we know that

$$
\frac{\mathrm{d}}{\mathrm{d} t} A_{\lambda_{n}}\left(x_{n}(t)\right)=A_{\lambda_{n}}^{\prime}\left(x_{n}(t)\right) x_{n}^{\prime}(t) \quad \text { a.e. on }[0, T]
$$

We return to (4.3) and use the above equality as well as hypothesis $\mathrm{H}_{0}$. So we obtain

$$
\left\langle V\left(x_{n}\right), \widehat{A}_{\lambda_{n}}\left(x_{n}\right)\right\rangle_{p p^{\prime}} \geq \int_{0}^{T}\left(\left\|x_{n}^{\prime}(t)\right\|_{\mathbb{R}^{N}}^{p-2} x_{n}^{\prime}(t), A_{\lambda_{n}}^{\prime}\left(x_{n}(t)\right) x_{n}^{\prime}(t)\right) \mathrm{d} t \geq 0
$$

Using this inequality in 4.2 , we obtain

$$
\left\|\widehat{A}_{\lambda_{n}}\left(x_{n}\right)\right\|_{2}^{2} \leq\left\|f_{n}\right\|_{2}\left\|\widehat{A}_{\lambda_{n}}\left(x_{n}\right)\right\|_{2}
$$

and so the sequence $\left\{\widehat{A}_{\lambda_{n}}\left(x_{n}\right)\right\}_{n \geq 1} \subseteq L^{2}\left([0, T] ; \mathbb{R}^{N}\right)$ is bounded. Thus we may assume that $\widehat{A}_{\lambda_{n}}\left(x_{n}\right) \longrightarrow \bar{u}$ weakly in $L^{2}\left([0, T] ; \mathbb{R}^{N}\right)$.

Arguing as in Claim 2 in the proof of Proposition 3 we can show that

$$
x_{n} \longrightarrow \bar{x} \text { in } W^{1, p}\left([0, T] ; \mathbb{R}^{N}\right)
$$

and

$$
\left\|x_{n}^{\prime}(\cdot)\right\|_{\mathbb{R}^{N}}^{p-2} x_{n}^{\prime}(\cdot) \longrightarrow\left\|\bar{x}^{\prime}(\cdot)\right\|_{\mathbb{R}^{N}}^{p-2} \bar{x}^{\prime}(\cdot) \quad \text { weakly in } W^{1, p^{\prime}}\left([0, T] ; \mathbb{R}^{N}\right) .
$$

Also, we have that

$$
\left\|f_{n}(t)\right\|_{\mathbb{R}^{N}} \leq a_{M}(t) \text { for a.a. } t \in[0, T],
$$

with $a_{M} \in L^{2}([0, T])$ (see hypothesis $\left.\mathrm{H}(\mathrm{F})_{1}(\mathrm{iii})\right)$ and so, passing to a subsequence if necessary, we may say that

$$
f_{n} \longrightarrow \bar{f} \quad \text { weakly in } L^{2}\left([0, T] ; \mathbb{R}^{N}\right),
$$


for some $\bar{f} \in L^{2}\left([0, T] ; \mathbb{R}^{N}\right)$. Using Proposition VII.3.9, p. 694 of [20], we have that

$$
\bar{f}(t) \in \overline{\text { conv }} \limsup _{n \rightarrow+\infty} F\left(t, x_{n}(t)\right) \subseteq F(t, \bar{x}(t)) \quad \text { a.e. on }[0, T]
$$

(the last inclusion is a consequence of hypothesis $\mathrm{H}(\mathrm{F})_{1}(\mathrm{ii})$ and of the fact that $F$ is $P_{k c}\left(\mathbb{R}^{N}\right)$-valued). So $\bar{f} \in S_{F(\cdot, \bar{x}(\cdot))}^{2}$. Therefore in the limit as $n \rightarrow+\infty$, we obtain

$$
\left(\left\|\bar{x}^{\prime}(t)\right\|_{\mathbb{R}^{N}}^{p-2} \bar{x}^{\prime}(t)\right)^{\prime}=\bar{u}(t)+\bar{f}(t) \quad \text { a.e. on }[0, T] .
$$

Since $\varphi\left(x_{n}^{\prime}(\cdot)\right) \longrightarrow \varphi\left(x^{\prime}(\cdot)\right)$ weakly in $W^{1, p^{\prime}}\left([0, T] ; \mathbb{R}^{N}\right)$, from the compactness of the embedding $W^{1, p^{\prime}}\left([0, T] ; \mathbb{R}^{N}\right) \subseteq C\left([0, T] ; \mathbb{R}^{N}\right)$, we have that

$$
\varphi\left(x_{n}^{\prime}(t)\right) \longrightarrow \varphi\left(\bar{x}^{\prime}(t)\right) \quad \forall t \in[0, T] .
$$

Because $\xi$ is maximal monotone, we have that $\mathrm{Gr} \xi \subseteq \mathbb{R}^{N} \times \mathbb{R}^{N}$ is closed. Since $\left(\varphi\left(x_{n}^{\prime}(0)\right),-\varphi\left(x_{n}^{\prime}(T)\right)\right) \in \xi\left(x_{n}(0), x_{n}(T)\right)$ for all $n \geq 1$, in the limit we have that

$$
\left(\varphi\left(\bar{x}^{\prime}(0)\right),-\varphi\left(\bar{x}^{\prime}(T)\right)\right) \in \xi(\bar{x}(0), \bar{x}(T)) .
$$

To show that $\bar{x} \in C^{1}\left([0, T] ; \mathbb{R}^{N}\right)$ is actually a solution of $[1.1$, we need to show that $\bar{u}(t) \in A(\bar{x}(t))$ almost everywhere on $[0, T]$. For this purpose, let $\widehat{J}_{\lambda_{n}}: L^{p}\left([0, T] ; \mathbb{R}^{N}\right) \longrightarrow$ $L^{p}\left([0, T] ; \mathbb{R}^{N}\right)$ be the Niemytzki operator corresponding to the map $J_{\lambda_{n}}: \mathbb{R}^{N} \longrightarrow \mathbb{R}^{N}$, i.e.

$$
\widehat{J}_{\lambda_{n}}(x)(\cdot)=J_{\lambda_{n}}(x(\cdot)) \quad \forall x \in L^{p}\left([0, T] ; \mathbb{R}^{N}\right) .
$$

Since $J_{\lambda_{n}}$ is nonexpansive, as before via the chain rule of Marcus and Mizel [25], we have that $\widehat{J}_{\lambda_{n}}\left(x_{n}\right) \in W^{1, p}\left([0, T] ; \mathbb{R}^{N}\right)$ and

$$
\frac{\mathrm{d}}{\mathrm{d} t} J_{\lambda_{n}}\left(x_{n}(t)\right)=J_{\lambda_{n}}^{\prime}\left(x_{n}(t)\right) x_{n}^{\prime}(t) \quad \text { a.e. on }[0, T],
$$

with

$$
\left\|J_{\lambda_{n}}^{\prime}\left(x_{n}(t)\right)\right\|_{\mathbb{R}^{N \times N}} \leq 1 \quad \text { a.e. on }[0, T] .
$$

So $\left\|J_{\lambda_{n}}^{\prime}\left(x_{n}(t)\right) x_{n}^{\prime}(t)\right\|_{\mathbb{R}^{N}} \leq\left\|x_{n}^{\prime}(t)\right\|_{\mathbb{R}^{N}}$ almost everywhere on $[0, T]$, from which we infer that the sequence $\left\{\widehat{J}_{\lambda_{n}}\left(x_{n}\right)\right\}_{n \geq 1} \subseteq W^{1, p}\left([0, T] ; \mathbb{R}^{N}\right)$ is bounded. Thus, passing to a next subsequence if necessary, we may assume that

$$
\begin{array}{ll}
\widehat{J}_{\lambda_{n}}\left(x_{n}\right) \longrightarrow v & \text { weakly in } W^{1, p}\left([0, T] ; \mathbb{R}^{N}\right), \\
\widehat{J}_{\lambda_{n}}\left(x_{n}\right) \longrightarrow v & \text { in } L^{p}\left([0, T] ; \mathbb{R}^{N}\right),
\end{array}
$$

for some $v \in W^{1, p}\left([0, T] ; \mathbb{R}^{N}\right)$. From the definition of the Yosida approximation, we have

$$
J_{\lambda_{n}}\left(x_{n}(t)\right)+\lambda_{n} A_{\lambda_{n}}\left(x_{n}(t)\right)=x_{n}(t) \quad \forall t \in[0, T] .
$$

So

$$
\widehat{J}_{\lambda_{n}}\left(x_{n}\right)+\lambda_{n} \widehat{A}_{\lambda_{n}}\left(x_{n}\right)=x_{n}
$$


Recall that $\left\{\widehat{A}_{\lambda_{n}}\left(x_{n}\right)\right\}_{n \geq 1} \subseteq L^{2}\left([0, T] ; \mathbb{R}^{N}\right)$ is bounded and $\lambda_{n} \searrow 0$. From 4.5. to the limit in (4.6), as $n \rightarrow+\infty$, we obtain $v=\bar{x}$. Therefore

$$
\begin{array}{ll}
\widehat{J}_{\lambda_{n}}\left(x_{n}\right) \longrightarrow \bar{x} & \text { weakly in } W^{1, p}\left([0, T] ; \mathbb{R}^{N}\right) \\
\widehat{J}_{\lambda_{n}}\left(x_{n}\right) \longrightarrow \bar{x} & \text { in } C\left([0, T] ; \mathbb{R}^{N}\right) .
\end{array}
$$

Let

$$
C \stackrel{d f}{=}\left\{t \in[0, T]: \exists(y, w) \in \operatorname{Gr} A \text { s.t. }(\bar{u}(t)-w, \bar{x}(t)-y)_{\mathbb{R}^{N}}<0\right\} .
$$

If we can show that $C$ is a Lebesgue-null set, then by virtue of the maximal monotonicity of $A$, we will have $\bar{u}(t) \in A(\bar{x}(t))$ almost everywhere on $[0, T]$.

Let

$$
\Gamma(t) \stackrel{d f}{=}\left\{(y, w) \in \operatorname{Gr} A:(\bar{u}(t)-w, \bar{x}(t)-y)_{\mathbb{R}^{N}}<0\right\} .
$$

Evidently $C=\{t \in[0, T]: \Gamma(t) \neq \emptyset\}$. Also

$$
\begin{aligned}
\operatorname{Gr} \Gamma= & ([0, T] \times \operatorname{Gr} A) \\
& \cap\left\{(t, y, w) \in[0, T] \times \mathbb{R}^{N} \times \mathbb{R}^{N}: \eta(t, y, w)<0\right\},
\end{aligned}
$$

where $\eta(t, y, w) \stackrel{d f}{=}(\bar{u}(t)-w, \bar{x}(t)-y)_{\mathbb{R}^{N}}$. Clearly the function $[0, T] \ni t \longmapsto \eta(t, y, w) \in \mathbb{R}$ is measurable and the function $\mathbb{R}^{N} \times \mathbb{R}^{N} \ni(y, w) \longmapsto \eta(t, y, w) \in \mathbb{R}$ is continuous. Hence $\eta$ is jointly measurable. Therefore

$$
\operatorname{Gr} \Gamma \in \mathscr{L}([0, T]) \times \mathscr{B}\left(\mathbb{R}^{N}\right) \times \mathscr{B}\left(\mathbb{R}^{N}\right)
$$

with $\mathscr{L}([0, T])$ being the Lebesgue $\sigma$-field of $[0, T]$. Invoking the Yankov-von NeumannAumann projection theorem (see [20], Theorem II.1.33, p. 149), we have

$$
\operatorname{proj}_{[0, T]} \operatorname{Gr} \Gamma=\{t \in[0, T]: \Gamma(t) \neq \emptyset\}=C \in \mathscr{L}([0, T]) .
$$

If $|C|>0$ (here by $|\cdot|$ we denote the Lebesgue measure on $[0, T]$ ), we can use the Yankovvon Neumann-Aumann selection theorem (see [20], Theorem II.2.14, p. 158), to obtain measurable functions $\bar{y}: C \longrightarrow \mathbb{R}^{N}$ and $\bar{w}: C \longrightarrow \mathbb{R}^{N}$, such that $(\bar{y}(t), \bar{w}(t)) \in \Gamma(t)$ for all $t \in C$. By virtue of Lusin's theorem, we can find a closed set $C_{1} \subseteq C$, such that $\left|C_{1}\right|>0$ and both restriction functions $\left.\bar{y}\right|_{C_{1}},\left.\bar{w}\right|_{C_{1}}$ are continuous and bounded. Since

$$
A_{\lambda_{n}}\left(x_{n}(t)\right) \in A\left(J_{\lambda_{n}}\left(x_{n}(t)\right)\right),
$$

we have

$$
\left(A_{\lambda_{n}}\left(x_{n}(t)\right)-\bar{w}(t), J_{\lambda_{n}}\left(x_{n}(t)\right)-\bar{y}(t)\right)_{\mathbb{R}^{N}} \geq 0 .
$$

So

$$
\int_{C_{1}}\left(A_{\lambda_{n}}\left(x_{n}(t)\right)-\bar{w}(t), J_{\lambda_{n}}\left(x_{n}(t)\right)-\bar{y}(t)\right)_{\mathbb{R}^{N}} \mathrm{~d} t \geq 0
$$

and thus, passing to the limit as $n \rightarrow+\infty$, we have

$$
\int_{C_{1}}(\bar{u}(t)-\bar{w}(t), \bar{x}(t)-\bar{y}(t))_{\mathbb{R}^{N}} \mathrm{~d} t \geq 0 .
$$


On the other hand, since $(\bar{y}(t), \bar{w}(t)) \in \Gamma(t)$, for all $t \in C$ and $\left|C_{1}\right|>0$, we have

$$
\int_{C_{1}}(\bar{u}(t)-\bar{w}(t), \bar{v}(t)-\bar{y}(t))_{\mathbb{R}^{N}} \mathrm{~d} t<0,
$$

we obtain a contradiction. This proves that $|C|=0$ and so $\bar{u}(t) \in A(\bar{x}(t))$ almost everywhere on $[0, T]$. Therefore $\bar{x} \in C^{1}\left([0, T] ; \mathbb{R}^{N}\right)$ is a solution of $[1.1$.

When $\operatorname{dom} A=\mathbb{R}^{N}$, we can slightly generalize the growth condition on $F$ by assuming that $a_{M} \in L^{p^{\prime}}([0, T])_{+}\left(1<p^{\prime} \leq 2\right)$ and drop hypothesis $\mathrm{H}_{0}$. Thus our hypotheses on $A$ and $F$ are the following:

$\mathrm{H}(\mathrm{A})_{2}: A: \mathbb{R}^{N} \longrightarrow 2^{\mathbb{R}^{N}}$ is a maximal monotone map, such that $\operatorname{dom} A=\mathbb{R}^{N}$ and $0 \in A(0)$.

$\mathrm{H}(\mathrm{F})_{2}: F:[0, T] \times \mathbb{R}^{N} \longrightarrow P_{k c}\left(\mathbb{R}^{N}\right)$ is a multifunction, which satisfies hypotheses $\mathrm{H}(\mathrm{F})_{1}$ (i), (ii), (iii), but with $a_{k} \in L^{p^{\prime}}([0, T])_{+}$and hypothesis $\mathrm{H}(\mathrm{F})_{1}$ (iv).

Theorem 2. If hypotheses $\mathrm{H}(\mathrm{A})_{2}, \mathrm{H}(\mathrm{F})_{2}$ and $\mathrm{H}(\xi)$ hold, then the solution set $S \subseteq$ $C^{1}\left([0, T] ; \mathbb{R}^{N}\right)$ of problem 1.1 is nonempty and closed.

Proof. Let $\lambda_{n} \searrow 0$ and let $x_{n} \in C^{1}\left([0, T] ; \mathbb{R}^{N}\right)$ be solutions of problem 3.1 (see Proposition 3 , note that the proposition is also valid under hypotheses $\mathrm{H}(\mathrm{F})_{2}$ instead of $\left.\mathrm{H}(\mathrm{F})_{1}\right)$. We know that

$$
\left\|x_{n}\right\|_{C\left([0, T] ; \mathbb{R}^{N}\right)} \leq M \quad \forall n \geq 1
$$

(see Claim 4 in the proof of Proposition 3. We have

$$
V\left(x_{n}\right)+\widehat{A}_{\lambda_{n}}\left(x_{n}\right)=-f_{n} \quad \text { with } f_{n} \in S_{F\left(\cdot, x_{n}(\cdot)\right)}^{p^{\prime}} .
$$

As in previous occasions, since $\widehat{A}_{\lambda_{n}}(0)=0$ and from Green's identity and hypothesis $\mathrm{H}(\xi)$, we have

$$
\left\|x_{n}^{\prime}\right\|_{p}^{p} \leq\left\langle V\left(x_{n}\right), x_{n}\right\rangle_{p p^{\prime}}+\left\langle\widehat{A}_{\lambda_{n}}\left(x_{n}\right), x_{n}\right\rangle_{p p^{\prime}}=-\left\langle f_{n}, x_{n}\right\rangle_{p p^{\prime}}
$$

So, from hypothesis $\mathrm{H}(\mathrm{F})_{2}(\mathrm{iii})$, we have that

$$
\left\|x_{n}^{\prime}\right\|_{p}^{p} \leq T^{1 / p} M\left\|a_{M}\right\|_{p^{\prime}} \quad \forall n \geq 1 .
$$

Thus the sequence $\left\{x_{n}^{\prime}\right\}_{n \geq 1} \subseteq L^{p}\left([0, T] ; \mathbb{R}^{N}\right)$ is bounded and so the sequence $\left\{x_{n}\right\}_{n \geq 1} \subseteq$ $W^{1, p}\left([0, T] ; \mathbb{R}^{N}\right)$ is also bounded.

Thus, by passing to a subsequence if necessary, we may assume that

$$
x_{n} \longrightarrow \bar{x} \quad \text { weakly in } W^{1, p}\left([0, T] ; \mathbb{R}^{N}\right)
$$

and

$$
x_{n} \longrightarrow \bar{x} \quad \text { in } L^{p}\left([0, T] ; \mathbb{R}^{N}\right)
$$

(recall that $W^{1, p}\left([0, T] ; \mathbb{R}^{N}\right)$ is embedded compactly in $L^{p}\left([0, T] ; \mathbb{R}^{N}\right)$ ). Recall that for all $n \geq 1$ and all $t \in[0, T]$, we have

$$
\left\|A_{\lambda_{n}}\left(x_{n}(t)\right)\right\|_{\mathbb{R}^{N}} \leq\left\|A^{0}\left(x_{n}(t)\right)\right\|_{\mathbb{R}^{N}} .
$$


Because $\operatorname{dom} A=\mathbb{R}^{N}$ (see [20], p. 307), we have that $A^{0}$ is bounded on compact sets. So, for all $t \in[0, T]$ and all $n \geq 1$, we have that

$$
\left\|A^{0}\left(x_{n}(t)\right)\right\|_{\mathbb{R}^{N}} \leq \sup \left\{\left\|A^{0}(\zeta)\right\|_{\mathbb{R}^{N}}: \zeta \in \bar{B}_{M}(0)\right\}<+\infty .
$$

Hence, we have that for all $t \in[0, T]$, the sequence $\left\{\left\|A_{\lambda_{n}}\left(x_{n}(t)\right)\right\|_{\mathbb{R}^{N}}\right\}_{n \geq 1}$ is uniformly bounded (i.e. it is bounded by a constant not depending on $n \geq 1$ and $t \in[0, T]$ ). Thus, also the sequence $\left\{\widehat{A}_{\lambda_{N}}\left(x_{n}\right)\right\}_{n \geq 1} \subseteq L^{p^{\prime}}\left([0, T] ; \mathbb{R}^{N}\right)$ is bounded and passing to a subsequence if necessary, we may assume that

$$
\widehat{A}_{\lambda_{n}}\left(x_{n}\right) \longrightarrow \bar{u} \quad \text { weakly in } L^{p^{\prime}}\left([0, T] ; \mathbb{R}^{N}\right) .
$$

As in the proof of Proposition 3 we can show that

$$
x_{n} \longrightarrow \bar{x} \text { in } W^{1, p}\left([0, T] ; \mathbb{R}^{N}\right)
$$

and

$$
\left\|x_{n}^{\prime}(\cdot)\right\|_{\mathbb{R}^{N}}^{p-2} \bar{x}_{n}^{\prime}(\cdot) \longrightarrow\left\|\bar{x}^{\prime}(\cdot)\right\|_{\mathbb{R}^{N}}^{p-2} \bar{x}^{\prime}(\cdot) \quad \text { in } W^{1, p^{\prime}}\left([0, T] ; \mathbb{R}^{N}\right) .
$$

Also, by virtue of hypothesis $\mathrm{H}(\mathrm{F})_{2}$ (iii), we may assume that

$$
f_{n} \longrightarrow \bar{f} \quad \text { weakly in } L^{p^{\prime}}\left([0, T] ; \mathbb{R}^{N}\right)
$$

and so as before, we can have that $\bar{f} \in S_{F(\cdot, x(\cdot))}^{p^{\prime}}$. So, in the limit as $n \rightarrow+\infty$, we have that

$$
\left(\left\|\bar{x}^{\prime}(t)\right\|_{\mathbb{R}^{N}}^{p-2} \bar{x}^{\prime}(t)\right)^{\prime}=\bar{u}(t)+\bar{f}(t) \quad \text { a.e. on }[0, T]
$$

Also, since $\varphi\left(x_{n}^{\prime}(\cdot)\right) \longrightarrow \varphi\left(x^{\prime}(\cdot)\right)$ weakly in $W^{1, p^{\prime}}\left([0, T] ; \mathbb{R}^{N}\right)$, we have that

$$
\varphi\left(\bar{x}^{\prime}(\cdot)\right) \longrightarrow \varphi\left(\bar{x}^{\prime}(\cdot)\right) \quad \text { in } C\left([0, T] ; \mathbb{R}^{N}\right)
$$

and so

$$
\varphi^{-1}\left(\left\|x_{n}^{\prime}(t)\right\|_{\mathbb{R}^{N}}^{p-2} x_{n}^{\prime}(t)\right)=x_{n}^{\prime}(t) \longrightarrow \bar{x}^{\prime}(t)=\varphi^{-1}\left(\left\|\bar{x}^{\prime}(t)\right\|_{\mathbb{R}^{N}}^{p-2} \bar{x}^{\prime}(t)\right),
$$

for all $t \in[0, T]$. Also recall that, at least for a subsequence, we have

$$
x_{n} \longrightarrow \bar{x} \quad \text { in } C\left([0, T] ; \mathbb{R}^{N}\right) .
$$

Since $\mathrm{Gr} \xi$ is closed in $\mathbb{R}^{N} \times \mathbb{R}^{N}$ and

$$
\left(\varphi\left(x_{n}^{\prime}(0)\right),-\varphi\left(x_{n}^{\prime}(T)\right)\right) \in \xi\left(x_{n}(0), x_{n}(T)\right) \quad \forall n \geq 1,
$$

in the limit as $n \rightarrow+\infty$, we obtain

$$
\left(\varphi\left(\bar{x}^{\prime}(0)\right),-\varphi\left(\bar{x}^{\prime}(T)\right)\right) \in \xi(\bar{x}(0), \bar{x}(T)) .
$$

Again, it remains to show that $\bar{u}(t) \in A(\bar{x}(t))$ almost everywhere on $[0, T]$. To this end, let $\widehat{A}: L^{p}\left([0, T] ; \mathbb{R}^{N}\right) \supseteq \widehat{D} \longrightarrow 2^{L^{p^{\prime}}\left([0, T] ; \mathbb{R}^{N}\right)}$, be defined by

$$
\widehat{A}(x) \stackrel{d f}{=}\left\{u \in L^{p^{\prime}}\left([0, T] ; \mathbb{R}^{N}\right): u(t) \in A(x(t)) \text { a.e. on }[0, T]\right\},
$$


for all $x \in \widehat{D} \stackrel{d f}{=}\left\{x \in L^{p}\left([0, T] ; \mathbb{R}^{N}\right): S_{A(x(\cdot))}^{p^{\prime}} \neq \emptyset\right\}$. Note that in particular, we have $C\left([0, T] ; \mathbb{R}^{N}\right) \subseteq \widehat{D}$.

We shall show that $\widehat{A}$ is maximal monotone. To this end, let $\varphi: \mathbb{R}^{N} \longrightarrow \mathbb{R}^{N}$ and $\widehat{\varphi}$ : $L^{p}\left([0, T] ; \mathbb{R}^{N}\right) \longrightarrow L^{p^{\prime}}\left([0, T] ; \mathbb{R}^{N}\right)$ be as in the proof of Proposition 3$]$ i.e. $\varphi(\zeta) \stackrel{d f}{=}\|\zeta\|_{\mathbb{R}^{N}}^{p-2} \zeta$ for $\zeta \in \mathbb{R}^{N}$ and $\widehat{\varphi}(x)(\cdot) \stackrel{d f}{=}\|x(\cdot)\|_{\mathbb{R}^{N}}^{p-2} x(\cdot)$ for $x \in L^{p}\left([0, T] ; \mathbb{R}^{N}\right)$. First, we show that $R(\widehat{A}+$ $\widehat{\varphi})=L^{p^{\prime}}\left([0, T] ; \mathbb{R}^{N}\right)$. For this purpose, take $h \in L^{p^{\prime}}\left([0, T] ; \mathbb{R}^{N}\right)$ and let

$$
\Gamma(t) \stackrel{d f}{=}\left\{(y, w) \in \mathbb{R}^{N} \times \mathbb{R}^{N}: w \in A(y), w+\varphi(y)=h(t),\|y\|_{\mathbb{R}^{N}} \leq r(t)\right\},
$$

where $r(t) \stackrel{d f}{=}\|h(t)\|_{\mathbb{R}^{N}}^{1 / p-1}+1$. Note that $A+\varphi$ is maximal monotone on $\mathbb{R}^{N}$ (see [20], Theorem III.3.3, p. 334) and so $\Gamma(t) \neq \emptyset$ almost everywhere on $[0, T]$ (see [20], proof of Theorem III.6.28, p. 371). We have

$$
\begin{aligned}
\operatorname{Gr} \Gamma= & \left\{(t, y, w) \in[0, T] \times \mathbb{R}^{N} \times \mathbb{R}^{N}:\right. \\
& \left.\eta(t, y, w)=0, d(w, A(y))=0,\|y\|_{\mathbb{R}^{N}} \leq r(t)\right\},
\end{aligned}
$$

where $\eta(t, y, w) \stackrel{d f}{=} w+\varphi(y)-h(t)$. Evidently $\eta$ is a Caratheodory function (i.e. measurable in $t \in[0, T]$ and continuous in $\left.(y, w) \in \mathbb{R}^{N} \times \mathbb{R}^{N}\right)$, thus it is jointly measurable. Also since $\mathbb{R}^{N} \ni \zeta \longmapsto A(\zeta) \in 2^{\mathbb{R}^{N}}$ is upper semicontinuous and $P_{k c}\left(\mathbb{R}^{N}\right)$-valued (because $\operatorname{dom} A=\mathbb{R}^{N}$; see [20], p. 365), we have that

$$
\mathbb{R}^{N} \times \mathbb{R}^{N} \ni(y, w) \longmapsto d(w, A(y)) \in \mathbb{R}_{+}
$$

is a lower semicontinuous function. Therefore

$$
\operatorname{Gr} \Gamma \in \mathscr{L}([0, T]) \times \mathscr{B}\left(\mathbb{R}^{N}\right) \times \mathscr{B}\left(\mathbb{R}^{N}\right),
$$

with $\mathscr{L}([0, T])$ being the Lebesgue $\sigma$-field on $[0, T]$. Invoking the Yankov-von NeumannAutmann selection theorem (see [20], Theorem II.2.14, p. 158), we obtain measurable maps $\bar{y}, \bar{w}:[0, T] \longrightarrow \mathbb{R}$, such that $(\bar{y}(t), \bar{w}(t)) \in \Gamma(t)$ for all $t \in[0, T]$. Evidently $\bar{y} \in$ $L^{p}\left([0, T] ; \mathbb{R}^{N}\right)$ and $\bar{w} \in L^{p^{\prime}}\left([0, T] ; \mathbb{R}^{N}\right)$. Hence

$$
R(\widehat{A}+\widehat{\varphi})=L^{p^{\prime}}\left([0, T] ; \mathbb{R}^{N}\right) .
$$

Now, we shall show that this surjectivity property implies the maximality of the monotone map $\widehat{A}$. Indeed, suppose that $\widetilde{x} \in L^{p}\left([0, T] ; \mathbb{R}^{N}\right), \widetilde{y} \in L^{p^{\prime}}\left([0, T] ; \mathbb{R}^{N}\right)$ and satisfy

$$
\langle\widehat{y}-\widetilde{y}, \widehat{x}-\widetilde{x}\rangle_{p p^{\prime}} \geq 0 \quad \forall \widehat{x} \in \widehat{D}, \widehat{y} \in \widehat{A}(x) .
$$

Because $R(\widehat{A}+\widehat{\varphi})=L^{p^{\prime}}\left([0, R] ; \mathbb{R}^{N}\right)$, we can find $x_{1} \in \widehat{D}$ and $y_{1} \in \widehat{A}\left(x_{1}\right)$, such that $\tilde{y}+$ $\widehat{\varphi}(\widetilde{x})=y_{1}+\widehat{\varphi}\left(x_{1}\right)$. Then

$$
\left\langle\widehat{y}-y_{1}-\widehat{\varphi}\left(x_{1}\right)+\widehat{\varphi}(\widetilde{x}), \widehat{x}-\widetilde{x}\right\rangle_{p p^{\prime}} \geq 0 \quad \forall \widehat{x} \in \widehat{D}, \widehat{y} \in \widehat{A}(x) .
$$

So, in particular, putting $\widehat{x}=x_{1}$ and $\widehat{y}=y_{1}$, we have

$$
\left\langle\widehat{\varphi}\left(x_{1}\right)-\widehat{\varphi}(\widetilde{x}), x_{1}-\widetilde{x}\right\rangle_{p p^{\prime}} \leq 0 \text {. }
$$


But clearly $\widehat{\varphi}$ is strictly monotone. Therefore, it follows that $\widetilde{x}=x_{1} \in \widehat{D}$ and $\widetilde{y}=y_{1} \in \widehat{A}\left(x_{1}\right)$, i.e. $(\widetilde{x}, \widetilde{y}) \in \mathrm{Gr} \widehat{A}$ and so $\widehat{A}$ is maximal monotone.

If $J_{\lambda_{n}}: \mathbb{R}^{N} \longrightarrow \mathbb{R}^{N}$ is the resolvent map of $A$, for all $t \in[0, T]$, we have

$$
\begin{aligned}
& \left\|J_{\lambda_{n}}\left(x_{n}(t)\right)-\bar{x}(t)\right\|_{\mathbb{R}^{N}} \\
& \quad \leq\left\|J_{\lambda_{n}}\left(x_{n}(t)\right)-J_{\lambda_{n}}(\bar{x}(t))\right\|_{\mathbb{R}^{N}}+\left\|J_{\lambda_{n}}(\bar{x}(t))-\bar{x}(t)\right\|_{\mathbb{R}^{N}} \\
& \quad \leq\left\|x_{n}(t)-\bar{x}(t)\right\|_{\mathbb{R}^{N}}+\left\|J_{\lambda_{n}}(\bar{x}(t))-\bar{x}(t)\right\|_{\mathbb{R}^{N}} .
\end{aligned}
$$

As the last two terms tend to zero, as $n \rightarrow+\infty$, we also have that

$$
\widehat{J}_{\lambda_{n}}\left(x_{n}\right) \longrightarrow \bar{x} \quad \text { in } L^{p}\left([0, T] ; \mathbb{R}^{N}\right)
$$

Recall that $A_{\lambda_{n}}\left(x_{n}(t)\right) \in A\left(J_{\lambda_{n}}\left(x_{n}(t)\right)\right)$ for all $t \in[0, T]$, and so

$$
\left(\widehat{J}_{\lambda_{n}}\left(x_{n}\right), \widehat{A}_{\lambda_{n}}\left(x_{n}\right)\right) \in \mathrm{Gr} \widehat{A} \quad \forall n \geq 1
$$

As $\widehat{A}$ is maximal monotone, it has a closed graph and thus, passing to the limit in the last inclusion as $n \rightarrow+\infty$, we obtain $(\bar{x}, \bar{u}) \in \mathrm{Gr} \widehat{A}$, i.e.

$$
\bar{u}(t) \in A(\bar{x}(t)) \quad \text { a.e. on }[0, T] .
$$

This proves that $\bar{x} \in C^{1}\left([0, T] ; \mathbb{R}^{N}\right)$ is a solution of $[1.1$, i.e. $S \neq \emptyset$.

Finally, we show that the solution set $S \subseteq C^{1}\left([0, T] ; \mathbb{R}^{N}\right)$ is closed. So, let $\left\{\bar{x}_{n}\right\}_{n \geq 1} \subseteq S$ be a sequence of solutions of (1.1) and assume that

$$
\bar{x}_{n} \longrightarrow \overline{\bar{x}} \quad \text { in } C^{1}\left([0, T] ; \mathbb{R}^{N}\right) \text {. }
$$

As before, we can show that

$$
\varphi\left(\bar{x}_{n}^{\prime}(\cdot)\right) \longrightarrow \varphi\left(\overline{\bar{x}}^{\prime}(\cdot)\right) \quad \text { weakly in } W^{1, p^{\prime}}\left([0, T] ; \mathbb{R}^{N}\right)
$$

Therefore, in the limit as $n \rightarrow+\infty$, we obtain that

$$
\left(\left\|\overline{\bar{x}}^{\prime}(t)\right\|_{\mathbb{R}^{N}}^{p-2} \overline{\bar{x}}^{\prime}(t)\right)^{\prime} \in A(\overline{\bar{x}}(t))+F(t, \overline{\bar{x}}(t)) \quad \text { a.e. on }[0, T] .
$$

Moreover, since $\mathrm{Gr} \xi$ is closed, we have that

$$
\left(\varphi\left(\overline{\bar{x}}^{\prime}(0)\right),-\varphi\left(\overline{\bar{x}}^{\prime}(T)\right)\right) \in \xi(\overline{\bar{x}}(0), \overline{\bar{x}}(T))
$$

i.e. $\overline{\bar{x}} \in S$.

Remark 2. If we strengthen the growth condition on $F$, to $\mathrm{H}(\mathrm{F})_{2}^{\prime}$ (iii) for almost all $t \in$ $[0, T]$, all $\zeta \in \mathbb{R}^{N}$ and all $u \in F(t, \zeta)$, we have $\|u\|_{\mathbb{R}^{N}} \leq a(t)+c\|\zeta\|_{\mathbb{R}^{N}}^{p-1}$, where $a \in$ $L^{p^{\prime}}([0, T])_{+}$and $c>0$. Then it is easy to check that the solution set $S \subseteq C^{1}\left([0, T] ; \mathbb{R}^{N}\right)$ is in fact compact.

We can have corresponding existence result for the 'nonconvex problem'. In this case our hypotheses on the multifunction $F(t, \zeta)$ are the following:

$\mathrm{H}(\mathrm{F})_{3}: F:[0, T] \times \mathbb{R}^{N} \longrightarrow P_{k}\left(\mathbb{R}^{N}\right)$ is a multifunction, such that 
(i) the multifunction $[0, T] \times \mathbb{R}^{N} \ni(t, \zeta) \longmapsto F(t, \zeta) \in 2^{\mathbb{R}}$ is graph measurable;

(ii) for almost all $t \in[0, T]$, the multifunction $\mathbb{R}^{N} \ni \zeta \longmapsto F(t, \zeta) \in 2^{\mathbb{R}^{N}}$ is lower semicontinuous;

(iii) for all $k>0$, there exists $a_{k} \in L^{2}([0, T])_{+}$, such that for almost all $t \in[0, T]$, all $\zeta \in \mathbb{R}^{N}$ with $\|\zeta\|_{\mathbb{R}^{N}} \leq k$ and all $u \in F(t, \zeta)$, we have $\|u\|_{\mathbb{R}^{N}} \leq a_{k}(t)$;

(iv) there exists $M>0$, such that for almost all $t \in[0, T]$, all $\zeta \in \mathbb{R}^{N}$, with $\|\zeta\|_{\mathbb{R}^{N}}=M$ and all $u \in F(t, \zeta)$, we have $(u, \zeta)_{\mathbb{R}^{N}} \geq 0$.

Theorem 3. If hypotheses $\mathrm{H}(\mathrm{A})_{1}, \mathrm{H}(\mathrm{F})_{3}, \mathrm{H}(\xi)$ and $\mathrm{H}_{0}$ hold, then problem (1.1) has a solution $\bar{x} \in C^{1}\left([0, T] ; \mathbb{R}^{N}\right)$.

Proof. As in the proof of Proposition 3 we consider the following modification of $F$ :

$$
F_{1}(t, \zeta) \stackrel{d f}{=}-F\left(t, p_{M}(\zeta)\right)+\varphi\left(p_{M}(\zeta)\right)
$$

Evidently $F_{1}$ is graph measurable, for almost all $t \in[0, T]$, the multifunction $\mathbb{R}^{N} \ni \zeta \longmapsto$ $F(t, \zeta) \in 2^{\mathbb{R}^{N}}$ is lower semicontinuous and for almost all $t \in[0, T]$, all $\zeta \in \mathbb{R}^{N}$ and all $u \in F_{1}(t, \zeta)$, we have that $\|u\|_{\mathbb{R}^{N}} \leq a_{M}(t)$. Consider the multivalued Niemytzki operator

$$
\widehat{F}_{1}: L^{p}\left([0, T] ; \mathbb{R}^{N}\right) \longrightarrow P_{f}\left(L^{p^{\prime}}\left([0, T] ; \mathbb{R}^{N}\right)\right)
$$

corresponding to $F_{1}$, i.e.

$$
\widehat{F}_{1}(x) \stackrel{d f}{=} S_{F_{1}(\cdot, x(\cdot))}^{p^{\prime}} \quad \forall x \in L^{p}\left([0, T] ; \mathbb{R}^{N}\right) .
$$

We show that $\widehat{F}_{1}$ is lower semicontinuous. To this end, it suffices to show that for every $h \in L^{p^{\prime}}\left([0, T] ; \mathbb{R}^{N}\right)$, the function

$$
L^{p}\left([0, T] ; \mathbb{R}^{N}\right) \ni x \longmapsto d\left(h, \widehat{F}_{1}(x)\right) \in \mathbb{R}_{+}
$$

is upper semicontinuous. Now, let $\vartheta \geq 0$ and let us consider the superlevel set

$$
U(\vartheta) \stackrel{d f}{=}\left\{x \in L^{p}\left([0, T] ; \mathbb{R}^{N}\right): d\left(h, \widehat{F}_{1}(x)\right) \geq \vartheta\right\} .
$$

We need to prove that $U(\vartheta)$ is closed. Let $\left\{x_{n}\right\}_{n \geq 1} \subseteq U(\vartheta)$ and assume that

$$
x_{n} \longrightarrow x \text { in } L^{p}\left([0, T] ; \mathbb{R}^{N}\right) .
$$

Passing to a subsequence if necessary, we may assume that

$$
x_{n}(t) \longrightarrow x(t) \text { for a.a. } t \in[0, T]
$$

and because $F_{1}(t, \cdot)$ is lower semicontinuous, so for almost all $t \in[0, T]$, we have that

$$
\limsup _{n \rightarrow+\infty} d\left(h(t), F_{1}\left(t, x_{n}(t)\right)\right) \leq d\left(h(t), F_{1}(t, x(t))\right) \quad \text { a.e. on }[0, T] .
$$

Also, by virtue of $\mathrm{Hu}$ and Papageorgiou ([20], p. 183) we have that

$$
\vartheta \leq d\left(h, \widehat{F}_{1}\left(x_{n}\right)\right)=\int_{0}^{T} d\left(h(t), F_{1}\left(t, x_{n}(t)\right)\right) \mathrm{d} t .
$$


So, using Fatou's lemma, we have

$$
\begin{aligned}
\vartheta & \leq \limsup _{n \rightarrow+\infty} d\left(h, \widehat{F}_{1}\left(x_{n}\right)\right)=\limsup _{n \rightarrow+\infty} \int_{0}^{T} d\left(h(t), F_{1}\left(t, x_{n}(t)\right)\right) \mathrm{d} t \\
& \leq \int_{0}^{T} \limsup _{n \rightarrow+\infty} d\left(h(t), F_{1}\left(t, x_{n}(t)\right)\right) \mathrm{d} t \\
& \leq \int_{0}^{T} d\left(h(t), F_{1}(t, x(t))\right) \mathrm{d} t=d\left(h, \widehat{F}_{1}(x)\right),
\end{aligned}
$$

and thus $x \in U(\vartheta)$, i.e., $U(\vartheta)$ is closed and so $\widehat{F}_{1}$ is lower semicontinuous.

Clearly, the values of $\widehat{F}_{1}$ are decomposable sets. So according to Theorem II.8.7, p. 245 of [20], there exists a continuous map

$$
u: L^{p}\left([0, T] ; \mathbb{R}^{N}\right) \longrightarrow L^{p^{\prime}}\left([0, T] ; \mathbb{R}^{N}\right),
$$

such that

$$
u(x) \in \widehat{F}_{1}(x) \quad \forall x \in L^{p}\left([0, T] ; \mathbb{R}^{N}\right) .
$$

We consider the following approximation to problem 1.1.

$$
\begin{cases}\left(\left\|x^{\prime}(t)\right\|_{\mathbb{R}^{N}}^{p-2} x^{\prime}(t)\right)^{\prime}=A_{\lambda}(x(t))+u(x)(t) & \text { a.e. on }[0, T], \\ \left(\varphi\left(x^{\prime}(0)\right),-\varphi\left(x^{\prime}(T)\right) \in \xi(x(0), x(T))\right) & \lambda>0 .\end{cases}
$$

Viewing $u$ as a function from $W^{1, p}\left([0, T] ; \mathbb{R}^{N}\right)$ into $L^{p^{\prime}}\left([0, T] ; \mathbb{R}^{N}\right)$, we see that problem (4.7) is equivalent to fixed point problem $x=K_{\lambda}^{-1} u(x)$, with

$$
K_{\lambda}: L^{p}\left([0, T] ; \mathbb{R}^{N}\right) \supseteq D \longrightarrow L^{p^{\prime}}\left([0, T] ; \mathbb{R}^{N}\right)
$$

as in the proof of Proposition 3 Arguing as in the proof of Proposition 3 using this time the classical single-valued Leray-Schauder alternative principle, we obtain a solution for problem 4.7).

Finally, if $\lambda_{n} \searrow 0$ and $x_{n} \in C^{1}\left([0, T] ; \mathbb{R}^{N}\right)$ for $n \geq 1$, are solutions of problem [4.7], with $\lambda=\lambda_{n}$, as in the proof of Theorem 1 we can show that

$$
x_{n} \longrightarrow \bar{x} \quad \text { in } C^{1}\left([0, T] ; \mathbb{R}^{N}\right)
$$

and $\bar{x}$ solves 1.1 .

Again, if $\operatorname{dom} A=\mathbb{R}^{N}$, we can slightly strengthen the growth condition on $F$ and obtain the nonconvex counterpart of Theorem 2

$\mathrm{H}(\mathrm{F})_{4}: F:[0, T] \times \mathbb{R}^{N} \longrightarrow P_{k}\left(\mathbb{R}^{N}\right)$ is a multifunction, which satisfies hypotheses $\mathrm{H}(\mathrm{F})_{3}$ (i), (ii), (iii), but with $a_{k} \in L^{p^{\prime}}([0, T])_{+}$and hypothesis $\mathrm{H}(\mathrm{F})_{1}$ (iv).

Theorem 4. If hypotheses $\mathrm{H}(\mathrm{A})_{2}, \mathrm{H}(\mathrm{F})_{4}$ and $\mathrm{H}(\xi)$ hold, then problem 1.1 has a solution $\bar{x} \in C^{1}\left([0, T] ; \mathbb{R}^{N}\right)$. 


\section{Special cases}

In this section, we indicate some special problems of interest which fit into our general framework and illustrate the generality and unifying character of our work here.

Example 1. Let $K_{1}, K_{2} \subseteq \mathbb{R}^{N}$ be nonempty, closed and convex sets with $0 \in K_{1} \cap K_{2}$. Let $\delta_{K_{1} \times K_{2}}$ be the indicator function of $K_{1} \times K_{2}$, i.e.

$$
\delta_{K_{1} \times K_{2}}\left(\zeta, \zeta^{\prime}\right)= \begin{cases}0 & \text { if }\left(\zeta, \zeta^{\prime}\right) \in K_{1} \times K_{2} \\ +\infty & \text { otherwise }\end{cases}
$$

Then, we know that $\delta_{K_{1} \times K_{2}}$ is a proper, convex and lower semicontinuous function on $\mathbb{R}^{N} \times \mathbb{R}^{N}$ and

$$
\partial \delta_{K_{1} \times K_{2}}=N_{K_{1} \times K_{2}}=N_{K_{1}} \times N_{K_{2}}
$$

(here, if $C \subseteq \mathbb{R}^{N}$, by $N_{C}$ we denote the normal cone to $C$; see e.g. [20], p. 534). Let $\xi=\partial \delta_{K_{1} \times K_{2}}$. Then problem 1.1 becomes

$$
\left\{\begin{array}{l}
\left(\left\|x^{\prime}(t)\right\|_{\mathbb{R}^{N}}^{p-2} x^{\prime}(t)\right)^{\prime} \in A(x(t))+F(t, x(t)) \quad \text { a.e. on }[0, T] \\
x(0) \in K_{1}, \quad x(T) \in K_{2}, \\
\left(x^{\prime}(0), x(0)\right)_{\mathbb{R}^{N}}=\sigma\left(x^{\prime}(0), K_{1}\right), \\
\left(-x^{\prime}(T), x(T)\right)_{\mathbb{R}^{N}}=\sigma\left(x^{\prime}(T), K_{2}\right) .
\end{array}\right.
$$

Note that, if $C \subseteq \mathbb{R}^{N}, \sigma(\cdot, C): \mathbb{R}^{N} \longrightarrow \bar{R}=\mathbb{R} \cup\{+\infty\}$ denotes the support function of $C$, i.e.

$$
\sigma(\zeta, C)=\sup \left\{(\zeta, c)_{\mathbb{R}^{N}}: c \in C\right\} .
$$

The map $\xi$ is maximal monotone, since it is the subdifferential of $\delta_{K_{1} \times K_{2}}$. Because $0 \in$ $K_{1} \cap K_{2}$, we have that $(0,0) \in \xi(0,0)$ and if $\left(b, b^{\prime}\right) \in \xi\left(a, a^{\prime}\right)=N_{K_{1}}(a) \times N_{K_{2}}\left(a^{\prime}\right)$, we have that $(b, a)_{\mathbb{R}^{N}} \geq 0$ and $\left(b^{\prime}, a^{\prime}\right)_{\mathbb{R}^{N}} \geq 0$, hence hypothesis $\mathrm{H}(\xi)$ is valid. Therefore the results of this paper apply to problem 5.1 .

Example 2. Let us take $K_{1}, K_{2} \subseteq \mathbb{R}_{+}^{N}$ as before, $\psi=\delta_{\mathbb{R}_{+}^{N}}$ and $A=\partial \psi$. For $\zeta=$ $\left(\zeta_{1}, \zeta_{2}, \ldots, \zeta_{N}\right) \in \mathbb{R}_{+}^{N}$, we have

$$
A(\zeta)=N_{\mathbb{R}_{+}^{N}}(\zeta) \stackrel{d f}{=}\left\{\begin{array}{llrl}
\{0\} & \text { if } \quad x_{k}>0 & \text { for all } k \in\{1,2, \ldots, N\}, \\
-\mathbb{R}_{+}^{N} \cap\{\zeta\}^{\perp} & \text { if } & x_{k}=0 & \text { for at least one, } \\
& & & k \in\{1,2, \ldots, N\} .
\end{array}\right.
$$

In this case, we check that for all $\lambda>0$, we have

$$
A_{\lambda}(\zeta)=\frac{1}{\lambda}\left(\zeta-p\left(\zeta ; \mathbb{R}_{+}^{N}\right)\right)
$$

with $p\left(\cdot ; \mathbb{R}_{+}^{N}\right)$ being the metric projection on $\mathbb{R}_{+}^{N}$. For $\zeta \in K_{1} \cup K_{2}$, we have $p\left(\zeta ; \mathbb{R}_{+}^{N}\right)=$ $\zeta$ and so $A_{\lambda}(\zeta)=0$. Thus the hypothesis $\mathrm{H}_{0}$ is satisfied. Problem 1.1 becomes the following evolutionary variational inequality: 


$$
\left\{\begin{array}{l}
\quad\left(\left\|x^{\prime}(t)\right\|_{\mathbb{R}^{N}}^{p-2} x^{\prime}(t)\right)^{\prime} \in F(t, x(t)) \\
\quad \text { a.e. on }\left\{t \in[0, T]: x_{k}(t)>0 \text { for all } k \in\{1,2, \ldots, N\}\right\}, \\
\quad\left(\left\|x^{\prime}(t)\right\|_{\mathbb{R}^{N}}^{p-2} x^{\prime}(t)\right)^{\prime} \in F(t, x(t))-u(t) \\
\quad \text { a.e. on }\left\{t \in[0, T]: x_{k}(t)=0 \text { for at least one } k \in\{1,2, \ldots, N\}\right\}, \\
x(0) \in K_{1}, x(T) \in K_{2}, \\
x(t) \in \mathbb{R}_{+}^{N}, u(t) \in \mathbb{R}_{+}^{N},(x(t), u(t))_{\mathbb{R}^{N}}=0 \quad \forall t \in[0, T], \\
\left(x^{\prime}(0), x(0)\right)_{\mathbb{R}^{N}}=\sigma\left(x^{\prime}(0), K_{1}\right), \\
\left(-x^{\prime}(T), x(T)\right)_{\mathbb{R}^{N}}=\sigma\left(-x^{\prime}(T), K_{2}\right) .
\end{array}\right.
$$

Example 3. If in the previous case, we choose $K_{1}=K_{2}=\{0\}$, then $N_{K_{1}}=N_{K_{2}}=\mathbb{R}^{N}$ and so there are no constraints on $x^{\prime}(0)$ and $x^{\prime}(T)$. Therefore, problem (1.1) becomes the classical Dirichlet problem. Moreover, since $A_{\lambda}(0)=0$, we see that hypothesis $\mathrm{H}_{0}$ holds.

Example 4. If $K_{1}=K_{2}=\mathbb{R}^{N}$, then $N_{K_{1}}=N_{K_{2}}=\{0\}$ and so there are no constraints on $x(0), x(T)$ and $x^{\prime}(0)=x^{\prime}(T)=0$. Thus problem (1.1) becomes the classical Neumann problem. Clearly hypothesis $\mathrm{H}_{0}$ is automatically satisfied.

The scalar (i.e. $N=1$ ) Neumann problem with $A \equiv 0$ was studied recently by Kandikakis and Papageorgiou [23], using a different approach.

Example 5. If

$$
K=\left\{\left(\zeta, \zeta^{\prime}\right) \in \mathbb{R}^{N} \times \mathbb{R}^{N}: \zeta=\zeta^{\prime}\right\}
$$

and

$$
\xi=\partial \delta_{K}=K^{\perp}=\left\{\left(\zeta, \zeta^{\prime}\right) \in \mathbb{R}^{N} \times \mathbb{R}^{N}: \zeta=-\zeta^{\prime}\right\},
$$

then problem 1.1 becomes the periodic problem. Again hypothesis $\mathrm{H}_{0}$ is automatically satisfied since

$$
\left(A_{\lambda}(a), a^{\prime}\right)_{\mathbb{R}^{N}}+\left(A_{\lambda}(d), d^{\prime}\right)_{\mathbb{R}^{N}}=0 \quad \forall\left(a^{\prime}, d^{\prime}\right) \in \xi(a, d) .
$$

The nonconvex periodic problem for first-order inclusions was studied recently by De Blasi et al [4], Hu et al [18] and $\mathrm{Hu}$ and Papageorgiou [19]. In their formulation $A \equiv 0$ and their methods are degree theoretic.

Example 6. Let $\xi: \mathbb{R}^{N} \times \mathbb{R}^{N} \longrightarrow \mathbb{R}^{N} \times \mathbb{R}^{N}$ be defined by

$$
\xi\left(\zeta, \zeta^{\prime}\right)=\left(\frac{1}{\vartheta^{p-1}} \varphi(\zeta), \frac{1}{\eta^{p-1}} \varphi\left(\zeta^{\prime}\right)\right)
$$

with $\vartheta, \eta>0$, then problem 1.1 becomes a problem of Sturm-Liouville type with boundary conditions

$$
x(0)-\vartheta x^{\prime}(0)=0, \quad x(T)+\eta x^{\prime}(T)=0 .
$$

It is easy to see that hypotheses $\mathrm{H}(\xi)(\mathrm{i})$ and $\mathrm{H}_{0}$ are satisfied. 


\section{References}

[1] Bader R, A topological fixed point theory for evolution inclusions, Zeitshrift für Anal. Anwend. 20 (2001) 3-15

[2] Boccardo L, Drabek P, Giachetti D and Kucera M, Generalizations of Fredholm alternative for nonlinear differential operators, Nonlinear Anal. 10 (1986) 1083-1103

[3] Dang H and Oppenheimer S F, Existence and uniqueness results for some nonlinear boundary value problems, J. Math. Anal. Appl. 198 (1996) 35-48, doi:10.1006/ jmaa.1996.0066

[4] De Blasi F S, Górniewicz L and Pianigiani G, Topological degree and periodic solutions of differential inclusions, Nonlinear Anal. 37 (1999) 217-245

[5] De Blasi F S and Pianigiani G, The Baire category method in existence problem for a class of multivalued equations with nonconvex right hand side, Funkcialaj Ekvacioj 28 (1985) 139-156

[6] De Blasi F S and Pianigiani G, Nonconvex valued differential inclusions in Banach spaces, J. Math. Anal. Appl. 157 (1991) 469-494

[7] De Blasi F S and Pianigiani G, On the density of extremal solutions of differential inclusions, Ann. Polonici Math. LVI (1992) 133-142

[8] De Blasi F S and Pianigiani G, Topological properties of nonconvex differential inclusions, Nonlinear Anal. 20 (1993) 871-894

[9] De Coster C, Pairs of positive solutions for the one-dimensional p-Laplacian, Nonlinear Anal. 23 (1994) 669-681

[10] Dugundji J and Granas A, Fixed point theory (Warsaw: Polish Scientific Publishers) (1982)

[11] Erbe L and Krawcewicz W, Nonlinear boundary value problems for differential inclusions $y^{\prime \prime} \in F\left(t, y, y^{\prime}\right)$, Annales Polonici Math. LIV (1991) 195-226

[12] Frigon M, Theoremes d'existence des solutions d'inclusion differentielles, NATO ASI Series, Section C, Vol. 472 (The Netherlands: Kluwer, Dordrecht) (1995) 51-87

[13] Gaines R and Mawhin J, Coincidence degree and nonlinear differential equations, Lecture Notes in Math. 568 (New York: Springer Verlag) (1977)

[14] Guo Z, Boundary value problems of a class of quasilinear ordinary differential equations, Diff. Integral Eqns 6 (1993) 705-719

[15] Halidias N and Papageorgiou N S, Existence and relaxation results for nonlinear second order multivalued boundary value problems in $\mathbb{R}^{N}$, J. Diff. Eqns 147 (1998) 123-154, doi:10.1006/jdeq.1998.3439

[16] Hartman P, On Boundary value problems for systems of ordinary nonlinear second order differential equations, Trans. AMS 96 (1960) 493-509

[17] Hartman P, Ordinary differential equations (New York: Wiley) (1964).

[18] Hu S, Kandilakis D and Papageorgiou N S, Periodic solutions for nonconvex differential inclusions, Proc. AMS 127 (1999) 89-94

[19] $\mathrm{Hu}$ S and Papageorgiou N S, On the existence of periodic solutions for nonconvexvalued differential inclusions in $\mathbb{R}^{N}$, Proc. AMS 123 (1995) 3043-3050

[20] Hu S and Papageorgiou N S, Handbook of multivalued analysis. Volume I: Theory (The Netherlands: Kluwer, Dordrecht) (1997)

[21] Hu S and Papageorgiou N S, Handbook of multivalued analysis. Volume II: Applications, (The Netherlands: Kluwer, Dordrecht) (2000)

[22] Kandilakis D and Papageorgiou N S, Existence theorem for nonlinear boundary value problems for second order differential inclusions, J. Diff. Eqns 132 (1996) 107-125, doi:10.1006/jdeq.1996.0173.

[23] Kandilakis D and Papageorgiou N S, Neumann problem for a class of quasilinear differential equations, Atti. Sem. Mat. Fisico Univ. di Modena 48 (1999) 1-15

[24] Knobloch W, On the existence of periodic solutions for second order vector differential 
equations, J. Diff. Eqns 9 (1971) 67-85

[25] Marcus M and Mizel V, Absolute continuity on tracks and mappings of Sobolev spaces, Arch. Rational Mech. Anal. 45 (1972) 294-320

[26] Mawhin J, Some boundary value problems for Hartman-type perturbations of the ordinary vector $p$-Laplacian, Nonlinear Anal. 40 (2000) 497-503 